\title{
Prescribing the center of mass of a multi-soliton solution for a perturbed semilinear wave equation
}

\author{
M.A. Hamza \\ Imam Abdulrahman Bin Faisal University P.O. Box 1982 Dammam, Saudi Arabia \\ Hatem Zaag \\ Université Paris 13, Sorbonne Paris Cité, \\ LAGA, CNRS (UMR 7539), F-93430, Villetaneuse, France
}

\begin{abstract}
We construct a finite-time blow-up solution for a class of strongly perturbed semilinear wave equation with an isolated characteristic point in one space dimension. Given any integer $k \geq 2$ and $\zeta_{0} \in \mathbb{R}$, we construct a blow-up solution with a characteristic point $a$, such that the asymptotic behavior of the solution near $(a, T(a))$ shows a decoupled sum of $k$ solitons with alternate signs, whose centers (in the hyperbolic geometry) have $\zeta_{0}$ as a center of mass, for all times. Although the result is similar to the unperturbed case in its statement, our method is new. Indeed, our perturbed equation is not invariant under the Lorentz transform, and this requires new ideas. In fact, the main difficulty in this paper is to prescribe the center of mass $\zeta_{0} \in \mathbb{R}$. We would like to mention that our method is valid also in the unperturbed case, and simplifies the original proof by Côte and Zaag [12, as far as the center of mass prescription is concerned.
\end{abstract}

MSC 2010 Classification: 35L05, 35L71, 35L67, 35B44, 35B40, 35B20.

Keywords: Semilinear wave equation, blow-up, one-dimensional case, characteristic point, multi-solitons, perturbations.

\section{Introduction}

This paper is concerned with blow-up solutions of a perturbed semilinear wave equation

$$
\left\{\begin{array}{l}
\partial_{t}^{2} u=\partial_{x}^{2} u+|u|^{p-1} u+f(u)+g\left(x, t, u, \partial_{x} u, \partial_{t} u\right) \\
u(0)=u_{0} \text { and } \partial_{t} u(0)=u_{1}
\end{array}\right.
$$

where $u(t): x \in \mathbb{R} \rightarrow u(x, t) \in \mathbb{R}, p>1, u_{0} \in \mathrm{H}_{\mathrm{loc}, \mathrm{u}}^{1}$ and $u_{1} \in \mathrm{L}_{\mathrm{loc}, \mathrm{u}}^{2}$ with $\|v\|_{\mathrm{L}_{\mathrm{loc}, \mathrm{u}}^{2}}^{2}=$ $\sup _{a \in \mathbb{R}} \int_{|x-a|<1}|v(x)|^{2} d x$ and $\|v\|_{\mathrm{H}_{\text {loc,u }}^{1}}^{2}=\|v\|_{\mathrm{L}_{\text {loc,u }}^{2}}^{2}+\|\nabla v\|_{\mathrm{L}_{\text {loc,u }}^{2}}^{2}$. We assume that $f$ and $g$ are $\mathscr{C}^{1}$ functions, where $f: \mathbb{R} \rightarrow \mathbb{R}$ and $g: \mathbb{R}^{4} \rightarrow \mathbb{R}$ satisfy the following conditions:

$$
(H) \begin{cases}|f(u)| \leq M_{0}\left(1+\frac{|u|^{p}}{\log \left(2+u^{2}\right)^{\alpha}}\right), & \text { for all } u \in \mathbb{R}, \\ |g(x, t, u, v, z)| \leq M_{0}\left(1+|u|^{\frac{p+1}{2}}+|v|+|z|\right), & \text { for all } x, t, u, v, z \in \mathbb{R} .\end{cases}
$$

where $M_{0}>0$ and $\alpha>1$. 
Under the more restrictive assumptions

$$
\left(H^{\prime}\right) \begin{cases}|f(u)| \leq M_{1}\left(1+|u|^{q}\right), & \text { for all } u \in \mathbb{R}, \\ |g(x, t, v, z)| \leq M_{1}(1+|v|+|z|), & \text { for all } x, t, v, z \in \mathbb{R} .\end{cases}
$$

where $M_{1}>0$ and $q<p$, we are able to add more information to our results (see Theorem 1 and Corollary 2).

As we will explain in details later, we would like to draw the attention of the reader to the fact that we are making two major steps in considering equation (11) under assumption $(H)$ in comparison with our previous papers dedicated to perturbations of the wave equation and written under assumption $\left(H^{\prime}\right)([28]$, [29], [30], [31])

- we allow a quasi critical perturbation $f(u)$ (in log scales), but this was already the case in ([25], [26], [27]).

- we allow a $u$ dependance in $g$, with a growth up to $|u|^{\frac{p+1}{2}}$.

The Cauchy problem for equation (1) is solved in the space $\mathrm{H}_{\text {loc,u }}^{1} \times \mathrm{L}_{\text {loc,u }}^{2}$. This follows from the finite speed of propagation and the well-posedness in $H^{1}(\mathbb{R}) \times L^{2}(\mathbb{R})$ (see for example Georgiev and Todorova [16]). The existence of blow-up solutions $u(t)$ of (11) follows from energy techniques (see for example Levine and Todorova [36] and Todorova [60]).

Note that in this paper, we consider a class of perturbations of the idealized equation (when $f \equiv g \equiv 0$ ). This is quite meaningful, since physical models are sometimes damped and hardly come with a pure power source term (see Whitham [61]). For more applications in general relativity, see Donninger, Schlag and Soffer [13].

If $u$ is an arbitrary blow-up solution of (11), we define (see for example Alinhac [1]) a 1-Lipschitz curve $\Gamma=\{(x, T(x))\}$ such that the maximal influence domain $D$ of $u$ (or the domain of definition of $u$ ) is written as

$$
D=\{(x, t) \mid t<T(x)\} .
$$

$\bar{T}=\inf _{x \in \mathbb{R}} T(x)$ and $\Gamma$ are called the blow-up time and the blow-up graph of $u$. A point $x_{0}$ is a non characteristic point if there are

$$
\delta_{0} \in(0,1) \text { and } t_{0}<T\left(x_{0}\right) \text { such that } u \text { is defined on } \mathscr{C}_{x_{0}, T\left(x_{0}\right), \delta_{0}} \cap\left\{t \geq t_{0}\right\}
$$

where $\mathscr{C}_{\bar{x}, \bar{t}, \bar{\delta}}=\{(x, t)|t<\bar{t}-\bar{\delta}| x-\bar{x} \mid\}$. We denote by $\mathscr{R}$ (resp. $\mathscr{S}$ ) the set of non characteristic (resp. characteristic) points.

In the case $(f, g) \equiv(0,0))$, equation (1) reduces to the semilinear wave equation:

$$
\left\{\begin{array}{l}
\partial_{t}^{2} u=\partial_{x}^{2} u+|u|^{p-1} u \\
u(0)=u_{0} \text { and } \partial_{t} u(0)=u_{1} .
\end{array}\right.
$$

In a series of papers [47, 48, [51] and [52] (see also the note [49]), Merle and Zaag together with Côte and Zaag [12] give a full picture of blow-up for solutions of equation (44) in one space dimension. Furthermore, in [12], for any integer $k \geq 2$ and $\zeta_{0} \in \mathbb{R}$, Côte and Zaag construct a blow-up solution with a characteristic point $a$, such that the asymptotic behavior of the solution near $(a, T(a))$ shows a decoupled sum of $k$ solitons 
with alternate signs, whose centers (in the hyperbolic geometry) have $\zeta_{0}$ as a center of mass, for all times. Let us note that the invariance of equation (44) under the Lorentz transform was crucial in [12] to prescribe the center of mass.

More generally, in [30, we also construct a finite-time blow-up solution with a characteristic point in the unperturbed case, under assupmtion $\left(H^{\prime}\right)$, showing multi-solitons and prescribing the center of mass, if $g \equiv 0$. However, when $g \not \equiv 0$, we were unable to prescribe the center of mass of the multi-soliton, since in this case equation (1) is not invariant under the Lorentz transform. That obstruction justifies our new paper, where we invent new ideas in order to prescribe the center of mass of the multi-soliton. More importantly, our method works under the more general assumption $(H)$. Moreover, this new method is of course valid also for equation (4). Even better, the prescription of the center of mass for (4) is easier thanks to our method.

Our aim in this work is to prescribe the center of mass of the multi-soliton. More precisely, for any integer $k \geq 2$ and $\zeta_{0} \in \mathbb{R}$, we construct a blow-up solution with a characteristic point $a$, such that the asymptotic behavior of the solution near $(a, T(a))$ shows a decoupled sum of $k$ solitons with alternate signs, whose centers (in the hyperbolic geometry) have $\zeta_{0}$ as a center of mass, for all times.

Before stating our result, let us introduce the following similarity variables, for any $\left(x_{0}, T_{0}\right)$ such that $0<T_{0} \leq T\left(x_{0}\right)$ :

$$
w_{x_{0}, T_{0}}(y, s)=\left(T_{0}-t\right)^{\frac{2}{p-1}} u(x, t), \quad y=\frac{x-x_{0}}{T_{0}-t}, \quad s=-\log \left(T_{0}-t\right) .
$$

If $T_{0}=T\left(x_{0}\right)$, we will simply write $w_{x_{0}}$ instead of $w_{x_{0}, T\left(x_{0}\right)}$. The function $w=w_{x_{0}}$ satisfies the following equation for all $y \in(-1,1)$ and $s \geq-\log T\left(x_{0}\right)$ :

$$
\begin{aligned}
\partial_{s}^{2} w= & \mathscr{L} w-\frac{2(p+1)}{(p-1)^{2}} w+|w|^{p-1} w-\frac{p+3}{p-1} \partial_{s} w-2 y \partial_{y, s}^{2} w+e^{-\frac{2 p s}{p-1}} f\left(e^{\frac{2 s}{p-1}} w\right) \\
& +e^{-\frac{2 p s}{p-1}} g\left(x_{0}+y e^{-s}, T_{0}-e^{-s}, e^{\frac{(p+1) s}{p-1}} \partial_{y} w, e^{\frac{(p+1) s}{p-1}}\left(\partial_{s} w+y \partial_{y} w+\frac{2}{p-1} w\right)\right)
\end{aligned}
$$

where $\mathscr{L} w=\frac{1}{\rho} \partial_{y}\left(\rho\left(1-y^{2}\right) \partial_{y} w\right)$ and $\rho=\rho(y)=\left(1-y^{2}\right)^{\frac{2}{p-1}}$.

In the unperturbed case where $f \equiv g \equiv 0$, the Lyapunov functional for equation (6)

$$
E(w(s))=\int_{-1}^{1}\left(\frac{1}{2}\left(\partial_{s} w\right)^{2}+\frac{1}{2}\left(\partial_{y} w\right)^{2}\left(1-y^{2}\right)+\frac{(p+1)}{(p-1)^{2}} w^{2}-\frac{1}{p+1}|w|^{p+1}\right) \rho d y
$$

is defined for $\left(w, \partial_{s} w\right) \in \mathscr{H}$ where

$$
\mathscr{H}=\left\{\left(q_{1}, q_{2}\right) \mid\left\|\left(q_{1}, q_{2}\right)\right\|_{\mathscr{H}}^{2} \equiv \int_{-1}^{1}\left(q_{1}^{2}+\left(q_{1}^{\prime}\right)^{2}\left(1-y^{2}\right)+q_{2}^{2}\right) \rho d y<+\infty\right\} .
$$

We also introduce the projection of the space $\mathscr{H}$ defined in (8) on the first coordinate:

$$
\mathscr{H}_{0}=\left\{r \in H_{l o c}^{1} \mid\|r\|_{\mathscr{H}_{0}}^{2} \equiv \int_{-1}^{1}\left(r^{2}+\left(r^{\prime}\right)^{2}\left(1-y^{2}\right)\right) \rho d y<+\infty\right\}
$$


Moreover, we introduce for all $|d|<1$ the following solitons defined by

$$
\kappa(d, y)=\kappa_{0} \frac{\left(1-d^{2}\right)^{\frac{1}{p-1}}}{(1+d y)^{\frac{2}{p-1}}} \text { where } \kappa_{0}=\left(\frac{2(p+1)}{(p-1)^{2}}\right)^{\frac{1}{p-1}} \text { and }|y|<1 .
$$

Note that $\kappa(d)$ is a stationary solution of (6) , in the particular case where $(f, g) \equiv(0,0)$. We also introduce

$$
\bar{\zeta}_{i}(s)=\left(i-\frac{(k+1)}{2}\right) \frac{(p-1)}{2} \log s+\bar{\alpha}_{i}(p, k)
$$

where the sequence $\left(\bar{\alpha}_{i}\right)_{i=1, \ldots, k}$ is uniquely determined by the fact that $\left(\bar{\zeta}_{i}(s)\right)_{i=1, \ldots, k}$ is an explicit solution with zero center of mass for the following ODE system:

$$
\frac{1}{c_{1}} \dot{\zeta}_{i}=e^{-\frac{2}{p-1}\left(\zeta_{i}-\zeta_{i-1}\right)}-e^{-\frac{2}{p-1}\left(\zeta_{i+1}-\zeta_{i}\right)}, \quad \forall i=1, \ldots, k .
$$

where $c_{1}=c_{1}(p)>0$ and $\zeta_{0}(s) \equiv \zeta_{k+1}(s) \equiv 0$ (see Section 2 in [12] for more details).

Given an arbitrary blow-up solution $u(x, t)$ to (10) and a characteristic point $x_{0}$, we could extend in [30] the results first proved for (44) by Merle and Zaag in [50], and showed the following results, under the assumption $\left(H^{\prime}\right)$ (and we think the results can be easily extended to assumption $(H)$ following our strategy in [30])

(Description of the behavior of $w_{x_{0}}$ where $x_{0}$ is characteristic) Consider $x_{0} \in \mathscr{S}$. Then, there is $\zeta_{0}\left(x_{0}\right) \in \mathbb{R}$ such that

$$
\left\|\left(\begin{array}{c}
w_{x_{0}}(s) \\
\partial_{s} w_{x_{0}}(s)
\end{array}\right)-\theta_{1}\left(\sum_{i=1}^{k\left(x_{0}\right)}(-1)^{i+1} \kappa\left(d_{i}(s)\right)\right)\right\|_{\mathscr{H}} \rightarrow 0
$$

as $s \rightarrow \infty$, for some $k\left(x_{0}\right) \geq 2, \theta_{1}= \pm 1$ and continuous $d_{i}(s)=-\tanh \zeta_{i}(s)$ with

$$
\zeta_{i}(s)=\bar{\zeta}_{i}(s)+\zeta_{0},
$$

where $\bar{\zeta}_{i}(s)$ is introduced above in (11).

Following this description result, we naturally ask the corresponding construction question:

Can we construct an example for each blow-up modality described in (13)?

In other words, given $k \geq 2$ and and $\zeta_{0} \in \mathbb{R}$, can we construct a solution to equation (11) showing the behavior (13) and (14)?

In [12, Côte and Zaag showed that this is possible in the unperturbed case (44). In [30], we had a partial result for equation (10), in the sense that we could prescribe the number of solitons, but not the location of their center of mass, unless $g \equiv 0$. Indeed, the method of [12] extends with no difficulty to equation (1), if $g \equiv 0$. If not, or if assumption $(H)$ holds, we need new ideas and this is the aim of the paper. More precisely, this is our main result: 
Theorem 1 (Existence of a solution to (1) with prescribed blow-up behavior at a characteristic point). Assume that assumption $(H)$ holds. For any integer $k \geq 2$ and $\zeta_{0} \in \mathbb{R}$, there exists a blow-up solution $u(x, t)$ to equation (1) in $\mathrm{H}_{\text {loc,u }}^{1} \times \mathrm{L}_{\text {loc, } \mathrm{u}}^{2}(\mathbb{R})$ such that

$$
\left\|\left(\begin{array}{c}
w_{0}(s) \\
\partial_{s} w_{0}(s)
\end{array}\right)-\left(\begin{array}{c}
\sum_{i=1}^{k}(-1)^{i+1} \kappa\left(d_{i}(s)\right) \\
0
\end{array}\right)\right\|_{\mathscr{H}} \rightarrow 0 \text { as } s \rightarrow \infty,
$$

with

$$
d_{i}(s)=-\tanh \zeta_{i}(s), \quad \zeta_{i}(s)=\bar{\zeta}_{i}(s)+\zeta_{0}
$$

and $\bar{\zeta}_{i}(s)$ defined in (11). Moreover, if $\left(H^{\prime}\right)$ holds, then the origin is a characteristic point (i.e. $0 \in \mathscr{S}$ ).

Remark. Note from (16) and (11) that the center of mass of $\zeta_{i}(s)$ is fixed, in the sense that

$$
\frac{\zeta_{1}(s)+\cdots+\zeta_{k}(s)}{k}=\frac{\bar{\zeta}_{1}(s)+\cdots+\bar{\zeta}_{k}(s)}{k}+\zeta_{0}=\zeta_{0}, \quad \forall s \geq-\log T(0) .
$$

Remark. Let us remark from the blow-up description we made in [30] that, under the assumption $\left(H^{\prime}\right)$ (and we think the results can be easily extended to assumption $(H)$ ), the origin is an isolated characteristic point. We also would like to mention that, as in the unperturbed case (4), under the assumption $(H)$ (even under assumption $\left(H^{\prime}\right)$ ) we are unable to say whether this solution has other characteristic points or not. In particular, we have been unable to find a solution with $\mathscr{S}$ exactly equal to $\{0\}$. Nevertheless, let us remark that from the finite speed of propagation, we can prescribe more characteristic points, as follows:

Corollary 2 (Prescribing more characteristic points). Assume that assumption $(H)$ holds. Let $I=\left\{1, \ldots, n_{0}\right\}$ or $I=\mathbb{N}$ and for all $n \in I, x_{n} \in \mathbb{R}, T_{n}>0, k_{n} \geq 2$ and $\zeta_{0, n} \in \mathbb{R}$ such that

$$
x_{n}+T_{n}<x_{n+1}-T_{n+1} .
$$

Then, there exists a blow-up solution $u(x, t)$ of equation (11) in $\mathrm{H}_{\text {loc,u }}^{1} \times \mathrm{L}_{\text {loc, } \mathrm{u}}^{2}(\mathbb{R})$ with $T\left(x_{n}\right)=T_{n}$ and for all $n \in I$,

$$
\left\|\left(\begin{array}{c}
w_{x_{n}}(s) \\
\partial_{s} w_{x_{n}}(s)
\end{array}\right)-\left(\begin{array}{c}
\sum_{i=1}^{k_{n}}(-1)^{i+1} \kappa\left(d_{i, n}(s)\right) \\
0
\end{array}\right)\right\|_{\mathscr{H}} \rightarrow 0 \text { as } s \rightarrow \infty,
$$

with

$$
\forall i=1, \ldots, k_{n}, \quad d_{i, n}(s)=-\tanh \zeta_{i, n}(s), \quad \zeta_{i, n}(s)=\bar{\zeta}_{i}(s)+\zeta_{0, n}
$$

and $\bar{\zeta}_{i}(s)$ defined in (11). Moreover, if $\left(H^{\prime}\right)$ holds, then $\left\{x_{n} \mid n \in I\right\} \subset \mathscr{S}$.

Remark. Again, we are unable to construct a solution with $\mathscr{S}=\left\{x_{n} \mid n \in I\right\}$.

As one can see from (15) and (16), the solution we have just constructed in Theorem 1 behaves like the sum of $k$ solitons as $s \rightarrow \infty$. In the literature, such a solution is called a multi-soliton solution. Constructing multi-soliton solutions is an important problem in nonlinear dispersive equations. It has already be done for the $L^{2}$ critical and subcritical nonlinear Schrödinger equation (NLS) (see Merle [42] and Martel and Merle [40]), the $L^{2}$ critical and subcritical generalized Korteweg de Vries equation (gKdV) (see Martel 
[39]), and for the $L^{2}$ supercritical case both for (gKdV) and (NLS) equations in Côte, Martel and Merle [11].

More generally, constructing a solution to some PDE with a prescribed behavior (not necessarily multi-solitons solutions) is an important question. That question was solved for $(\mathrm{gKdV})$ by Côte [9, 10], and also for parabolic equations exhibiting blow-up, like the semilinear heat equation by Bressan [3, 4] (with an exponential source), Merle [43, Bricmont and Kupiainen [5, Merle and Zaag in [46, 45, Schweyer [57] (in the critical case), Mahmoudi, Nouaili and Zaag 38, (in the periodic case), the complex GinzburgLandau equation by Zaag [62, Masmoudi and Zaag in [41] and also Nouaili and Zaag [55], a complex heat equation with no gradient structure by Nouaili and Zaag [54], a gradient perturbed heat equation in the subcritical case by Ebde and Zaag in [15], then by Tayachi and Zaag in the critical case in [58, 59] and also by Ghoul, Nguyen and Zaag in [19], a strongly perturbed heat equation in Nguyen and Zaag [53], a non scaling invariant heat equation in Duong, Nguyen, and Zaag [14], two non variational parabolic system Ghoul, Nguyen and Zaag [20, 21, 22] or a higher order parabolic equation in [23]. Other examples are available for Schrödinger maps (see Merle, Raphaël and Rodnianski [44]), the wave maps (see Ghoul, Ibrahim and Nguyen [17]), and also for the Keller-Segel model (see Raphaël and Schweyer [56], and also Ghoul and Masmoudi [18]).

Surprisingly enough, in both the parabolic equations above and the supercritical dispersive equations treated in [11, the same topological argument is crucial to control the directions of instability. This will be the case again for the semilinear wave equation (11) under consideration. As for the strategy of the proof, we rely on the following two step method:

- As in the unperturbed case, thanks to a dynamical system formulation, we show that controlling the similarity variables version $w(y, s)$ solution of (6) around the expected behavior (15) reduces to the control of the unstable directions, whose number is finite. This dynamical system formulation is essentially the same as the one used in [12] and [52.

- Then, we solve the finite dimensional problem thanks to a topological argument based on index theory.

We would like to insist on the fact that we introduce a new feature in the method to allow us to directly prescribe the center of mass as required in (17).

\section{Construction of a multi-soliton solution in similarity vari- ables}

In this section, we assume that $(H)$ holds, and we construct a multi-soliton solution in similarity variables for equation (6). At the end of the section, we use this construction to prove Theorem 1 (note that we don't prove Corollary 2, as it follows immediately from Theorem 1 thanks to the finite speed of propagation). At the very end of the section, we will consider the more restrictive hypothesis $\left(H^{\prime}\right)$ to show that the origin is a characteristic point.

Technically, we use the dynamical system formulation introduced in 52 and used in [12]. For that purpose, we introduce for all $d \in(-1,1)$ and $\nu>-1+|d|, \kappa^{*}(d, \nu, y)=$ 
$\left(\kappa_{1}^{*}, \kappa_{2}^{*}\right)(d, \nu, y)$ where

$$
\begin{aligned}
\kappa_{1}^{*}(d, \nu, y) & =\kappa_{0} \frac{\left(1-d^{2}\right)^{\frac{1}{p-1}}}{(1+d y+\nu)^{\frac{2}{p-1}}} \\
\kappa_{2}^{*}(d, \nu, y) & =\nu \partial_{\nu} \kappa_{1}^{*}(d, \nu, y)=-\frac{2 \kappa_{0} \nu}{p-1} \frac{\left(1-d^{2}\right)^{\frac{1}{p-1}}}{(1+d y+\nu)^{\frac{p+1}{p-1}}} .
\end{aligned}
$$

We refer to these functions as "generalized solitons" or solitons for short. Notice that for any $\mu \in \mathbb{R}, \kappa^{*}\left(d, \mu e^{s}, y\right)$ is a solution to equation (6) in the unperturbed case. Then, note that:

- $\kappa^{*}\left(d, \mu e^{s}, y\right) \rightarrow(\kappa(d), 0)$ in $\mathscr{H}$ as $s \rightarrow-\infty$;

- when $\mu=0$, we recover the stationary solutions $(\kappa(d), 0)$ defined in (10);

- when $\mu>0$, the solution exists for all $(y, s) \in(-1,1) \times \mathbb{R}$ and converges to 0 in $\mathscr{H}$ as $s \rightarrow \infty$ (it is a heteroclinic connection between $(\kappa(d), 0)$ and 0 );

- when $\mu<0$, the solution exists for all $(y, s) \in(-1,1) \times\left(-\infty, \log \left(\frac{|d|-1}{\mu}\right)\right)$ and blows up at time $s=\log \left(\frac{|d|-1}{\mu}\right)$.

We also introduce for $l=0$ or 1 , for any $d \in(-1,1)$ and $r \in \mathscr{H}$,

$$
\begin{gathered}
\Pi_{l}^{d}(r)=\phi\left(W_{l}(d), r\right), \quad \text { where } \\
\phi(q, r):=\int_{-1}^{1}\left(q_{1} r_{1}+q_{1}^{\prime} r_{1}^{\prime}\left(1-y^{2}\right)+q_{2} r_{2}\right) \rho d y, \\
W_{l}(d, y):=\left(W_{l, 1}(d, y), W_{l, 2}(d, y)\right), \quad \text { with }
\end{gathered}
$$

$$
W_{1,2}(d, y)(y)=c_{1}(d) \frac{\left(1-d^{2}\right)^{\frac{1}{p-1}}\left(1-y^{2}\right)}{(1+d y)^{\frac{2}{p-1}+1}}, \quad W_{0,2}(d, y)=c_{0} \frac{\left(1-d^{2}\right)^{\frac{1}{p-1}}(y+d)}{(1+d y)^{\frac{2}{p-1}+1}},
$$

for some positive $c_{1}(d)$ and $c_{0}$, and $W_{l, 1}(d, y) \in \mathscr{H}_{0}$ is uniquely determined as the solution of

$$
-\mathscr{L} r+r=\left(l-\frac{p+3}{p-1}\right) W_{l, 2}(d)-2 y \partial_{y} W_{l, 2}(d)+\frac{8}{p-1} \frac{W_{l, 2}(d)}{1-y^{2}}
$$

normalized by the fact that $\Pi_{l}^{d}\left(F_{l}(d)\right)=\phi\left(W_{l}(d), F_{l}(d)\right)$, where

$$
F_{1}(d, y)=\left(1-d^{2}\right)^{\frac{p}{p-1}}\left(\begin{array}{c}
\frac{1}{(1+d y)^{\frac{2}{p-1}+1}} \\
\frac{1}{(1+d y)^{\frac{2}{p-1}+1}}
\end{array}\right), \quad F_{0}(d, y)=\left(1-d^{2}\right)^{\frac{1}{p-1}}\left(\begin{array}{c}
\frac{y+d}{(1+d y)^{\frac{2}{p-1}+1}} \\
0
\end{array}\right)
$$

(see estimate (3.57) in [51] for more details).

Given $k \geq 2, \zeta_{0} \in \mathbb{R}$ and $s_{0}>0$, we will construct the multi-solution as a solution to the Cauchy problem of equation (6) with initial data (at $s=s_{0}$ ) depending on $k+1$ parameters: $\left|\nu_{i, 0}\right| \leq s_{0}^{-\frac{1}{2}-\left|\gamma_{i}\right|}$ for $i=1, \ldots, k$, and $\left|\phi_{1,0}\right| \leq s_{0}^{-\eta}$, given by

$$
\left(w\left(y, s_{0}\right), \partial_{s} w\left(y, s_{0}\right)\right)=\sum_{i=1}^{k}(-1)^{i} \kappa^{*}\left(\hat{d}_{i}\left(s_{0}, \phi_{1,0}\right), \nu_{i, 0}, y\right),
$$

where

$$
\hat{d}_{i}\left(s_{0}, \phi_{1,0}\right)=-\tanh \left(\bar{\zeta}_{i}\left(s_{0}\right)+\zeta_{0}+\frac{p-1}{2} \phi_{1,0}\right),
$$


$\bar{\zeta}_{i}\left(s_{0}\right)$ is defined in (11), $\gamma_{i}$ is defined by

$$
\gamma_{i}=(p-1)\left(-i+\frac{k+1}{2}\right),
$$

and the constant $\eta>0$ will be defined later in (45).

In comparison with the unperturbed case treated in [12], we have one more parameter here $\left(\phi_{1,0}\right)$, and this parameter is precisely the one that will allow us to prescribe the center of mass. Note also that in [12, the corresponding construction was done with $\zeta_{0}=0$. Accordingly estimate (24) in [12] was satisfied with $\zeta_{0}=0$ and $\phi_{1,0}=0$. Here lays a major difference between our approach and that of [12], in the sense that we construct our solution in relation with $\left(\bar{\zeta}_{i}\left(s_{0}\right)+\zeta_{0}\right)_{i}$, which is a particular solution of (12), whereas in [12], the construction is done only for $\zeta_{0}=0$.

Such a solution will be denoted by $w\left(s_{0},\left(\nu_{i, 0}\right)_{i}, \phi_{1,0}, y, s\right)$, or when there is no ambiguity, by $w(y, s)$ or $w(s)$ for short. We will show that when $s_{0}$ is fixed large enough, we can fine-tune the parameters

$$
\nu_{i, 0} \in\left[-s_{0}^{-\frac{1}{2}-\left|\gamma_{i}\right|}, s_{0}^{-\frac{1}{2}-\left|\gamma_{i}\right|}\right] \quad(\text { for } \quad i=1, \ldots, k) \quad \text { and } \quad \phi_{1,0} \in\left[-s_{0}^{-\eta}, s_{0}^{-\eta}\right],
$$

so that the solution $w\left(s_{0},\left(\nu_{i, 0}\right)_{i}, \phi_{1,0}, y, s\right)$ will be decomposed as a sum of $k$ decoupled solitons. This is the aim of the section.

Proposition 2.1 (A multi-soliton solution in the $w(y, s)$ setting). For any integer $k \geq 2$, and $\zeta_{0} \in \mathbb{R}$ there exist $s_{0}>0, \nu_{i, 0} \in \mathbb{R}$ for $i=1, \ldots, k$ and $\phi_{1,0} \in \mathbb{R}$ such that equation (6) with initial data (at $\left.s=s_{0}\right)$ given by (23) is defined for all $(y, s) \in(-1,1) \times\left[s_{0}, \infty\right)$, satisfies $\left(w(s), \partial_{s} w(s)\right) \in \mathscr{H}$ for all $s \geq s_{0}$, and

$$
\left\|\left(\begin{array}{c}
w(s) \\
\partial_{s} w(s)
\end{array}\right)-\left(\begin{array}{c}
\sum_{i=1}^{k}(-1)^{i+1} \kappa\left(d_{i}(s)\right) \\
0
\end{array}\right)\right\|_{\mathscr{H}} \rightarrow 0 \text { as } s \rightarrow \infty,
$$

for some continuous $d_{i}(s)=-\tanh \zeta_{i}(s)$ satisfying

$$
\zeta_{i}(s)-\bar{\zeta}_{i}(s) \rightarrow \zeta_{0} \text { as } s \rightarrow \infty \text { for } i=1, \ldots, k
$$

where the $\bar{\zeta}_{i}(s)$ are introduced in (11).

Remark. Note from (23) that initial data are in $H^{1} \times L^{2}(-1,1)$. Going back to the $u(x, t)$ formulation, we see that initial data is also in $H^{1} \times L^{2}(-1,1)$ of the initial section of the backward light-cone. Therefore, from the solution to the Cauchy-problem in light-cones, we see that the solution stays in $H^{1} \times L^{2}$ of any section.

As one can see from (23), at the initial time $s=s_{0}, w\left(y, s_{0}\right)$ is a pure sum of solitons. From the continuity of the flow associated with equation (6) in $\mathscr{H}$ (this continuity comes from the continuity of the flow associated with equation (11) in $H^{1} \times L^{2}$ of sections of backward light-cones), $w(y, s)$ will stay close to a sum of solitons, at least for a short time after $s_{0}$. In fact, we can do better, and impose some orthogonality conditions, killing the zero and expanding directions of the linearized operator of equation (11) around the sum of solitons. The following modulation technique from Merle and Zaag in [52] is crucial for that. 
Proposition 2.2 (A modulation technique; Proposition 3.1 of [52]). For all $A \geq 1$, there exist $E_{0}(A)>0$ and $\epsilon_{0}(A)>0$ such that for all $E \geq E_{0}$ and $\epsilon \leq \epsilon_{0}$, if $v \in \mathscr{H}$ and for all $i=1, \ldots, k,\left(\hat{d}_{i}, \hat{\nu}_{i}\right) \in(-1,1) \times \mathbb{R}$ are such that

$$
-1+\frac{1}{A} \leq \frac{\hat{\nu}_{i}}{1-\left|\hat{d}_{i}\right|} \leq A, \quad \hat{\zeta}_{i+1}^{*}-\hat{\zeta}_{i}^{*} \geq E \text { and }\|\hat{q}\|_{\mathscr{H}} \leq \epsilon
$$

where $\hat{q}=v-\sum_{j=1}^{k}(-1)^{j} \kappa^{*}\left(\hat{d}_{j}, \hat{\nu}_{j}\right)$ and $\hat{d}_{i}^{*}=\frac{\hat{d}_{i}}{1+\hat{\nu}_{i}}=-\tanh \hat{\zeta}_{i}^{*}$, then, there exist $\left(d_{i}, \nu_{i}\right)$ such that for all $i=1, \ldots, k$ and $l=0,1$,

1. $\Pi_{l}^{d_{i}^{*}}(q)=0$ where $q:=v-\sum_{j=1}^{k}(-1)^{j} \kappa^{*}\left(d_{j}, \nu_{j}\right)$,

2. $\left|\frac{\nu_{i}}{1-\left|d_{i}\right|}-\frac{\hat{\nu}_{i}}{1-\left|\hat{d}_{i}\right|}\right|+\left|\zeta_{i}^{*}-\hat{\zeta}_{i}^{*}\right| \leq C(A)\|\hat{q}\|_{\mathscr{H}} \leq C(A) \epsilon$,

3. $-1+\frac{1}{2 A} \leq \frac{\nu_{i}}{1-\left|d_{i}\right|} \leq A+1, \zeta_{i+1}^{*}-\zeta_{i}^{*} \geq \frac{E}{2}$ and $\|q\|_{\mathscr{H}} \leq C(A) \epsilon$,

where $d_{i}^{*}=\frac{d_{i}}{1+\nu_{i}}=-\tanh \zeta_{i}^{*}$.

Let us apply this proposition with $v=\left(w\left(y, s_{0}\right), \partial_{s} w\left(y, s_{0}\right)\right)$ defined in (23), $\hat{d}_{i}=$ $\hat{d}_{i}\left(s_{0}, \phi_{1,0}\right)$ defined in (24) and $\hat{\nu}_{i}=\nu_{i, 0}$. Clearly, we have $\hat{q}=0$. Then, from (23), (11) and straightforward calculations, we see that

$$
\frac{\left|\hat{\nu}_{i}\right|}{1-\left|\hat{d}_{i}\right|} \leq \frac{C}{\sqrt{s_{0}}} \quad \text { and } \quad \hat{\zeta}_{i+1}^{*}-\hat{\zeta}_{i}^{*} \geq \frac{p-1}{4} \log s_{0}
$$

for $s_{0}$ large enough. Therefore, Proposition 2.2 applies with $A=2$ and from the continuity of the flow associated with equation (6) in $\mathscr{H}$, we have a maximal $\bar{s}=\bar{s}\left(s_{0},\left(\nu_{i, 0}\right)_{i}, \phi_{1,0}\right)>$ $s_{0}$ such that $w$ exists for all time $s \in\left[s_{0}, \bar{s}\right)$ and $w$ can be modulated in the sense that

$$
\left(w(y, s), \partial_{s} w(y, s)\right)=\sum_{i=1}^{k}(-1)^{i} \kappa^{*}\left(d_{i}(s), \nu_{i}(s)\right)+q(y, s)
$$

where the parameters $d_{i}(s)$ and $\nu_{i}(s)$ are such that for all $s \in\left[s_{0}, \bar{s}\right]$,

$$
\Pi_{l}^{d_{i}^{*}(s)}(q(s))=0, \quad \forall l=0,1, \quad i=1, \ldots, k
$$

and

$$
\frac{\left|\nu_{i}(s)\right|}{1-\left|d_{i}(s)\right|} \leq s_{0}^{-1 / 4}, \quad \zeta_{i+1}^{*}(s)-\zeta_{i}^{*}(s) \geq \frac{(p-1)}{8} \log s_{0} \quad \text { and } \quad\|q(s)\|_{\mathscr{H}} \leq \frac{1}{\sqrt{s_{0}}}
$$

where $\zeta_{i}^{*}(s)=-\arg \tanh \left(\frac{d_{i}(s)}{1+\nu_{i}(s)}\right)$.

Two cases then arise:

- either $\bar{s}\left(s_{0},\left(\nu_{i, 0}\right)_{i}, \phi_{1,0}\right)=+\infty$;

- or $\bar{s}\left(s_{0},\left(\nu_{i, 0}\right)_{i}, \phi_{1,0}\right)<+\infty$ and one of the $\leq$ symbols in (29) is a $=$. 
At this stage, we see that controlling the solution $w(s) \in \mathscr{H}$ is equivalent to controlling $q \in \mathscr{H},\left(d_{i}(s)\right)_{i} \in(-1,1)^{k}$ and $\left(\nu_{i}(s)\right)_{i} \in \mathbb{R}^{k}$. Introducing

$$
\zeta_{i}(s)=-\arg \tanh \left(d_{i}(s)\right), \quad J=\sum_{i=1}^{k-1} e^{-\frac{2}{p-1}\left(\zeta_{i+1}-\zeta_{i}\right)}, \quad \bar{J}=\sum_{i=1}^{k} \frac{\left|\nu_{i}\right|}{1-d_{i}^{2}},
$$

we project equation (6) to derive the following estimates:

Proposition 2.3 (Dynamics of the parameters). There exists $\delta>0$ such that for $s_{0}$ large enough and for all $s \in\left[s_{0}, \bar{s}\right)$, we have

$$
\begin{gathered}
\frac{\left|\dot{\nu}_{i}-\nu_{i}\right|}{1-d_{i}^{2}} \leq C\left(\|q\|_{\mathscr{H}}^{2}+J+\|q\|_{\mathscr{H}} \bar{J}\right)+\frac{C}{s^{\alpha}} \\
\left|\frac{\dot{\zeta}_{i}}{c_{1}}-\left(e^{-\frac{2}{p-1}\left(\zeta_{i}-\zeta_{i-1}\right)}-e^{-\frac{2}{p-1}\left(\zeta_{i+1}-\zeta_{i}\right)}\right)\right| \leq C\left(\|q\|_{\mathscr{H}}^{2}+(J+\|q\| \mathscr{H}) \bar{J}+J^{1+\delta}\right)+\frac{C}{s^{\alpha}}, \\
\|q(s)\|_{\mathscr{H}}^{2} \leq C e^{-\delta\left(s-s_{0}\right)}\left\|q\left(s_{0}\right)\right\|_{\mathscr{H}}^{2}+C J^{\bar{p}}+\frac{C}{s^{\alpha}}
\end{gathered}
$$

with

$$
\bar{p}= \begin{cases}p & \text { if } p<2, \\ 2-1 / 100 & \text { if } p=2, \\ 2 & \text { if } p>2,\end{cases}
$$

where $\zeta_{i}(s)=-\arg \tanh d_{i}(s), c_{1}=c_{1}(p)>0, J$ and $\bar{J}$, are introduced in (30).

Proof. As in the unperturbed case, this statement is devoted to understanding the dynamics of equation (6) near the sum of $k$ solitons. Most of the estimates are the same as in the unperturbed case (44) treated in [12] and [52] and some others are more delicate. For that reason, we leave the proof to Appendix $\mathrm{A}$.

Remark. Let us mention that, the estimates (31) (32) and (33) are similar to the ones obtained in the unperturbed case treated in [12] except for the following:

- The presence of the additional term $\frac{C}{s^{\alpha}}$ which is natural to control the perturbation terms related to $f$ and $g$.

- We end up, in estimate (3ㅏ) , we have a new term $J^{\bar{p}}$ instead of $\hat{J}^{2}$ (where $\hat{J}=\sum_{i=1}^{k-1} e^{-\frac{\bar{p}}{p-1}\left(\zeta_{i+1}-\zeta_{i}\right)}$ ) in the unperturbed case. This is based on the fact that all the norms on $\mathbb{R}^{n}$ are equivalent.

In order to prove Proposition 2.1. our aim is to show the existence of a solution with $\|q(s)\|_{\mathscr{H}} \rightarrow 0, \zeta_{i}(s)-\bar{\zeta}_{i}(s)-\zeta_{0} \rightarrow 0$ and $\bar{J}(s) \rightarrow 0$ as $s \rightarrow \infty$. Hence, it is natural to do as in Section 2 in [12] and linearize system (32) around $\left(\bar{\zeta}_{i}(s)+\zeta_{0}\right)_{i}$ by introducing

$$
\xi_{i}(s)=\frac{2}{p-1}\left(\zeta_{i}(s)-\bar{\zeta}_{i}(s)-\zeta_{0}\right) .
$$

This is reasonable, since we see from (32) and (29) that $\left(\zeta_{i}(s)\right)_{i}$ satisfies a perturbed version of the system (12) satisfied by $\left(\bar{\zeta}_{i}(s)+\zeta_{0}\right)_{i}$ and studied in [12]. 
Following (35)), if $\boldsymbol{\xi}(s)=\left(\xi_{1}(s), \ldots, \xi_{k}(s)\right)$, then, as it was done in the unperturbed case in [12], by writing a Taylor expansion of (32), we obtain the following differential inequalities, for all $s \in\left[s_{0}, \bar{s}\right)$, and $i=1, \ldots, k$,

$$
\left|\dot{\boldsymbol{\xi}}-\frac{1}{s} M \boldsymbol{\xi}\right| \leq \frac{C}{s}|\boldsymbol{\xi}|^{2}+C\left(\|q\|_{\mathscr{H}}^{2}+\left(J+\|q\|_{\mathscr{H}}\right) \bar{J}+J^{1+\delta}\right)+\frac{C}{s^{\alpha}},
$$

where the self-adjoint $k \times k$ matrix $M=\left(m_{i, j}\right)_{(i, j) \in \llbracket 1, k \rrbracket}$ is defined by

$$
m_{i, i-1}=\sigma_{i-1}, \quad m_{i, i}=-\left(\sigma_{i-1}+\sigma_{i}\right), \quad m_{i, i+1}=\sigma_{i}, \quad m_{i, j}=0 \text { if }|i-j| \geq 2,
$$

with

$$
\sigma_{i}=\frac{i(k-i)}{2} .
$$

Note that the matrix $M$ is diagonalizable, with real eigenvalues $\left(-m_{i}\right)_{i}$, defined by:

$$
-m_{i} \equiv-\frac{i(i-1)}{2}, \text { for } i=1, \ldots, k
$$

and the associated eigenvectors $\boldsymbol{e}_{i}$ normalized for the $\ell^{\infty}$ norm. Note that the kernel of $M$ is spanned by the vector

$$
e_{1}={ }^{t}(1, \ldots, 1)
$$

It is then natural to work in the basis defined by its eigenvectors $\left(\boldsymbol{e}_{i}\right)_{i}$ and to introduce $\phi(s)=\left(\phi_{1}(s), \ldots, \phi_{k}(s)\right)$ defined by

$$
\boldsymbol{\xi}(s)=\sum_{i=1}^{k} \phi_{i}(s) \boldsymbol{e}_{i}
$$

Note that if we project the differential inequalities (36) on the eigenfunctions $\left(\boldsymbol{e}_{i}\right)_{i}$ of $M$, then we trivially obtain the following:

Corollary 2.4 (Dynamics for $\phi_{i}$ ). For all $s \in\left[s_{0}, \bar{s}\right)$, and $i=1, \ldots, k$,

$$
\left|\dot{\phi}_{i}+\frac{m_{i}}{s} \phi_{i}\right| \leq \frac{C}{s} \sum_{j=1}^{k} \phi_{j}^{2}+C\left(\|q\|_{\mathscr{H}}^{2}+\left(J+\|q\|_{\mathscr{H}}\right) \bar{J}+J^{1+\delta}\right)+\frac{C}{s^{\alpha}} .
$$

Remark. This corollary is trivial. Indeed, we just write the differential inequalities (36) on the basis $\left(\boldsymbol{e}_{i}\right)_{i}$. Note that in [12], the authors prove the following sharper version of (42):

$$
\left|\dot{\phi}_{i}+\frac{m_{i}}{s} \phi_{i}\right| \leq \frac{C}{s} \sum_{j=2}^{k} \phi_{j}^{2}+C\left(\|q\|_{\mathscr{H}}^{2}+\left(J+\|q\|_{\mathscr{H}}\right) \bar{J}+J^{1+\delta}\right)+\frac{C}{s^{\alpha}} .
$$

Note that in (43), the index runs from 2 to $k$, and not from 1 to $k$ like in (42). That improvement was crucial in [12], since it was required that $\phi_{i}(s) \rightarrow 0$ as $s \rightarrow \infty$, only for $i \geq 2$, and not for $\phi_{1}(s)$. By the way, the proof of (43) is far from being easy. Here, since we work with any $\zeta_{0} \in \mathbb{R}$ (see (24)) and aim at prescribing the center of mass, we don't need to be that accurate, and from this point of view, our proof is more simple than the proof of [12]. 
Note that thanks to all these changes of variables, controlling $w$ is equivalent to the control of $\left(q, \phi,\left(\nu_{i}\right)_{i}\right)$. Now, in order to control $w$ near multi-solitons, we introduce the following set:

Definition 2.5 (Definition of a shrinking set for the parameters). We say that $w(s) \in$ $\mathscr{V}(s)$ if and only if

$$
\begin{aligned}
& s^{1 / 2+\eta}\|q\|_{\mathscr{H}} \leq 1, \\
& \quad \forall i=1, \ldots, k, \quad s^{1 / 2+\left|\gamma_{i}\right|}\left|\nu_{i}\right| \leq 1, \quad \text { and } \quad s^{\eta}\left|\phi_{i}\right| \leq 1,
\end{aligned}
$$

where

$$
\eta=\frac{1}{4} \min \left\{1, \delta, \frac{\bar{p}}{2}-\frac{1}{2}, \frac{\alpha-1}{2}\right\}
$$

$\delta>0$ is defined in Proposition 2.3 and $\bar{p}$ is defined in (34).

Remark. In [12, the condition $s^{\eta}\left|\phi_{i}(s)\right| \leq 1$ was required only for $i \geq 2$. When $i=1$, the approach of [12] requires only the smallness of $\phi_{1}(s)$ (namely that $\left|\phi_{1}(s)\right| \leq s_{0}^{-\eta}$ with $s_{0}$ and not $s$ ) with no need to have $\phi_{1}(s) \rightarrow 0$ as $s \rightarrow \infty$. Here, we will get $\phi_{1}(s) \rightarrow 0$ as $s \rightarrow \infty$, which is the key to prescribe the center of mass.

From the existence of $\bar{s}$ (defined right before (28) ), we know that there is a maximal $s^{*}\left(s_{0},\left(\nu_{i, 0}\right)_{i}, \phi_{1,0}\right) \in\left[s_{0}, \bar{s}\right)$ such that for all $s \in\left[s_{0}, s^{*}\right), w(s) \in \mathscr{V}(s)$ and:

- either $s^{*}=+\infty$,

- or $s^{*}<+\infty$ and from continuity, $w\left(s^{*}\right) \in \partial \mathscr{V}\left(s^{*}\right)$, in the sense that one $\leq$ symbol in (44) has to be replaced by the $=$ symbol. in

Our aim is to show that for $s_{0}$ large enough, one can find a parameter $\left(\left(\nu_{i, 0}\right)_{i}, \phi_{1,0}\right)$

$$
\prod_{i=1}^{k}\left[-s_{0}^{-\frac{1}{2}-\left|\gamma_{i}\right|}, s_{0}^{-\frac{1}{2}-\left|\gamma_{i}\right|}\right] \times\left[-s_{0}^{-\eta}, s_{0}^{-\eta}\right]
$$

such that

$$
s^{*}\left(s_{0},\left(\nu_{i, 0}\right)_{i}, \phi_{1,0}\right)=+\infty .
$$

With Proposition 2.3 and Corollary 2.4 at hand, we are in a position to prove the following, which directly implies Proposition 2.1.

Proposition 2.6 (A solution $w(y, s) \in \mathscr{V}(s)$ ). For $s_{0}$ large enough, there exists

$$
\left(\nu_{i, 0}\right)_{i} \in \prod_{i=1}^{k}\left[-s_{0}^{-\frac{1}{2}-\left|\gamma_{i}\right|}, s_{0}^{-\frac{1}{2}-\left|\gamma_{i}\right|}\right] \quad \text { and } \quad \phi_{1,0} \in\left[-s_{0}^{-\eta}, s_{0}^{-\eta}\right]
$$

such that equation (6) with initial data (at $s=s_{0}$ ) given by (23) is defined for all $(y, s) \in(-1,1) \times\left[s_{0}, \infty\right)$ and satisfies $w(s) \in \mathscr{V}(s)$ for all $s \geq s_{0}$.

Proof of Proposition 2.6. Let $s_{0}$ be large enough. Define $\mathbb{B}$ (resp. $\mathbb{S}$ ) the unit ball (resp. sphere) in $\left(\mathbb{R}^{k+1}, \ell^{\infty}\right)$, and the rescaling function

$$
\Gamma_{s}:{ }^{t}\left(\boldsymbol{\nu}_{\mathbf{1}}, \ldots, \boldsymbol{\nu}_{\boldsymbol{k}}, \boldsymbol{\phi}_{\mathbf{1}, \mathbf{0}}\right) \mapsto{ }^{t}\left(s^{-1 / 2-\left|\gamma_{1}\right|} \boldsymbol{\nu}_{\mathbf{1}}, \ldots, s^{-1 / 2-\left|\gamma_{k}\right|} \boldsymbol{\nu}_{\boldsymbol{k}}, s^{-\eta} \boldsymbol{\phi}_{\mathbf{1}, \mathbf{0}}\right) .
$$


For all $\left(\left(\boldsymbol{\nu}_{\boldsymbol{i}}\right)_{i}, \boldsymbol{\phi}_{\mathbf{1}, \mathbf{0}}\right) f \in \mathbb{B}$, we consider the solution $w\left(s_{0},\left(\boldsymbol{\nu}_{\boldsymbol{i}}\right)_{i}, \boldsymbol{\phi}_{\mathbf{1}, \mathbf{0}}, y, s\right)$ (or $w(y, s)$ for short) to equation (6), with initial condition at time $s_{0}$ given by (23) with

$$
{ }^{t}\left(\left(\nu_{i, 0}\right)_{i}, \phi_{1,0}\right)=\Gamma_{s_{0}}\left({ }^{t}\left(\left(\boldsymbol{\nu}_{\boldsymbol{i}}\right)_{i}, \boldsymbol{\phi}_{\mathbf{1 , 0}}\right)\right) .
$$

As we showed after the statement of Proposition 2.2, $w(y, s)$ can be modulated (up to some time $\left.\bar{s}=\bar{s}\left(s_{0},\left(\boldsymbol{\nu}_{\boldsymbol{i}}\right)_{i}, \boldsymbol{\phi}_{\mathbf{1}, \mathbf{0}}\right)>s_{0}\right)$ into a triplet $\left(q(s),\left(d_{i}(s)\right)_{i},\left(\nu_{i}(s)\right)_{i}\right)$. From the uniqueness of such a decomposition (which is a consequence of the application of the implicit function theorem, see the proof of [52, Proposition 3.1]), we get

$q\left(s_{0}\right)=0, \quad d_{i}\left(s_{0}\right)=\hat{d}_{i}\left(s_{0}, \phi_{1,0}\right) \quad$ and $\quad \nu_{i}\left(s_{0}\right)=\left[\Gamma_{s_{0}}\left({ }^{t}\left(\left(\boldsymbol{\nu}_{\boldsymbol{i}}\right)_{i}, \boldsymbol{\phi}_{\mathbf{1}, \mathbf{0}}\right)\right)\right]_{i}, \quad$ for $i=1, \ldots, k$,

where $\hat{d}_{i}\left(s_{0}, \phi_{1,0}\right)$ is defined in (24) .

From (48), the definitions (35) of $\xi_{i}(s)$ and (24) of $\hat{d}_{i}\left(s_{0}, \phi_{1,0}\right)$, we see that

$$
\forall i=1, \ldots, k, \quad \xi_{i}\left(s_{0}\right)=\phi_{1,0} .
$$

In other words, $\left(\xi_{i}\left(s_{0}\right)\right)_{i}$ is in the kernel of the matrix $M$ defined in (37) (see (40)). In particular

$$
\forall i=2, \ldots, k, \quad \phi_{i}\left(s_{0}\right)=0 \quad \text { and } \quad \phi_{1}\left(s_{0}\right)=\phi_{1,0} .
$$

Performing the change of variables (35) and (41), we reduce the control of $w(s)$ to the control of $\left(q(s),\left(\nu_{i}(s)\right)_{i},\left(\phi_{i}(s)\right)_{i}\right)$. Defining

$$
N\left(\left(\boldsymbol{\nu}_{\boldsymbol{i}}\right)_{i}, \boldsymbol{\phi}_{\mathbf{1}, \mathbf{0}}, s\right):=\max \left\{s^{1 / 2+\eta}\|q(s)\| \mathscr{H}_{i \geq 1}, \sup _{i \geq 1} s^{1 / 2+\left|\gamma_{i}\right|}\left|\nu_{i}(s)\right|, \sup _{i \geq 1} s^{\eta}\left|\phi_{i}(s)\right|\right\}
$$

we see that $\mathscr{V}(s)$ (Definition introduced in 2.5) is simply the unit ball of the norm $N\left(\left(\boldsymbol{\nu}_{\boldsymbol{i}}\right)_{i}, \boldsymbol{\phi}_{\mathbf{1}, \mathbf{0}}, s\right)$.

As asserted just before (46), we aim at finding $\left(\left(\boldsymbol{\nu}_{\boldsymbol{i}}\right)_{i}, \boldsymbol{\phi}_{\mathbf{1}, \mathbf{0}}\right)$ so that the associated solution of equation (6) $w \in \mathscr{C}\left(\left[s_{0}, \infty\right), \mathscr{H}\right)$ is globally defined for forward times and

$$
\forall s \geq s_{0}, \quad N\left(\left(\boldsymbol{\nu}_{\boldsymbol{i}}\right)_{i}, \boldsymbol{\phi}_{\mathbf{1}, \mathbf{0}}, s\right) \leq 1, \quad \text { i.e. } \quad w(s) \in \mathscr{V}(s) .
$$

We argue by contradiction. Assume that the conclusion of Proposition 2.6 does not hold. In particular, for all $\left(\left(\boldsymbol{\nu}_{\boldsymbol{i}}\right)_{i}, \boldsymbol{\phi}_{\mathbf{1}, \mathbf{0}}\right)$, the exit time $s^{*}\left(s_{0},\left(\boldsymbol{\nu}_{\boldsymbol{i}}\right)_{i}, \boldsymbol{\phi}_{\mathbf{1 , 0}}\right)$ is finite, where

$$
s^{*}\left(s_{0},\left(\boldsymbol{\nu}_{\boldsymbol{i}}\right)_{i}, \boldsymbol{\phi}_{\mathbf{1}, \mathbf{0}}\right)=\sup \left\{s \geq s_{0} \mid \forall \tau \in\left[s_{0}, s\right], N\left(\left(\boldsymbol{\nu}_{\boldsymbol{i}}\right)_{i}, \boldsymbol{\phi}_{\mathbf{1 , 0},}, \tau\right) \leq 1\right\} .
$$

Then, by continuity, notice that

$$
N\left(\left(\boldsymbol{\nu}_{\boldsymbol{i}}\right)_{i}, \boldsymbol{\phi}_{\mathbf{1}, \mathbf{0}}, s^{*}\left(s_{0},\left(\boldsymbol{\nu}_{\boldsymbol{i}}\right)_{i}, \boldsymbol{\phi}_{\mathbf{1}, \mathbf{0}}\right)\right)=1,
$$

and that the supremum defining $s^{*}\left(s_{0},\left(\boldsymbol{\nu}_{\boldsymbol{i}}\right)_{i}, \boldsymbol{\phi}_{\mathbf{1}, \mathbf{0}}\right)$ is in fact a maximum.

We now consider the (rescaled) flow for the $\left(\boldsymbol{\nu}_{\boldsymbol{i}}\right)_{i}$, that is

$$
\Phi:\left(s,\left(\boldsymbol{\nu}_{\boldsymbol{i}}\right)_{i}, \boldsymbol{\phi}_{\mathbf{1}, \mathbf{0}}\right) \mapsto \Gamma_{s}^{-1}\left({ }^{t}\left(\nu_{1}(s), \ldots, \nu_{k}(s), \phi_{1}(s)\right)\right) .
$$

By the properties of the flow, $\Phi$ is a continuous function of $\left(s,\left(\boldsymbol{\nu}_{\boldsymbol{i}}\right)_{i}, \boldsymbol{\phi}_{\mathbf{1 , 0}}\right) \in\left[s_{0}, s^{*}\left(s_{0},\left(\boldsymbol{\nu}_{\boldsymbol{i}}\right)_{i}, \boldsymbol{\phi}_{\mathbf{1 , 0}}\right)\right] \times$ $\mathbb{B}$. By definition of the exit time $s^{*}\left(s_{0},\left(\boldsymbol{\nu}_{\boldsymbol{i}}\right)_{i}, \boldsymbol{\phi}_{\mathbf{1}, \mathbf{0}}\right)$, we have that for all $s \in\left[s_{0}, s^{*}\left(s_{0},\left(\boldsymbol{\nu}_{\boldsymbol{i}}\right)_{i}, \boldsymbol{\phi}_{\mathbf{1}, \mathbf{0}}\right)\right]$, $\Phi\left(s,\left(\boldsymbol{\nu}_{\boldsymbol{i}}\right)_{i}, \boldsymbol{\phi}_{\mathbf{1}, \mathbf{0}}\right) \in \mathbb{B}$. The following claim allows us to conclude: 
Claim 2.7. For $s_{0}$ large enough, we have:

(i) For all $\left(\left(\boldsymbol{\nu}_{\boldsymbol{i}}\right)_{i}, \boldsymbol{\phi}_{\mathbf{1}, \mathbf{0}}\right) \in \mathbb{B}, \Phi\left(s^{*}\left(s_{0},\left(\boldsymbol{\nu}_{\boldsymbol{i}}\right)_{i}, \boldsymbol{\phi}_{\mathbf{1}, \mathbf{0}}\right),\left(\boldsymbol{\nu}_{\boldsymbol{i}}\right)_{i}, \boldsymbol{\phi}_{\mathbf{1}, \mathbf{0}}\right) \in \mathbb{S}$.

(ii) The flow $s \mapsto \Phi\left(s,\left(\boldsymbol{\nu}_{\boldsymbol{i}}\right)_{i}, \boldsymbol{\phi}_{\mathbf{1}, \mathbf{0}}\right)$ is transverse (outgoing) at $s=s^{*}\left(s_{0},\left(\boldsymbol{\nu}_{\boldsymbol{i}}\right)_{i}, \boldsymbol{\phi}_{\mathbf{1}, \mathbf{0}}\right)$ (when it hits $\mathbb{\$}$ ).

(iii) If $\left(\left(\boldsymbol{\nu}_{\boldsymbol{i}}\right)_{i}, \boldsymbol{\phi}_{\mathbf{1}, \mathbf{0}}\right) \in \mathbb{S}$, then $s^{*}\left(s_{0},\left(\boldsymbol{\nu}_{\boldsymbol{i}}\right)_{i}, \boldsymbol{\phi}_{\mathbf{1}, \mathbf{0}}\right)=s_{0}$ and $\Phi\left(s^{*}\left(s_{0},\left(\boldsymbol{\nu}_{\boldsymbol{i}}\right)_{i}, \boldsymbol{\phi}_{\mathbf{1}, \mathbf{0}}\right),\left(\boldsymbol{\nu}_{\boldsymbol{i}}\right)_{i}, \boldsymbol{\phi}_{\mathbf{1}, \mathbf{0}}\right)=$ $\left(\left(\nu_{i}\right)_{i}, \phi_{1,0}\right)$.

Proof of Claim 2.7. In the following, the constant $C$ stands for $C\left(s_{0}\right)$.

(i) Since for all $s \in\left[s_{0}, s^{*}\left(s_{0},\left(\boldsymbol{\nu}_{\boldsymbol{i}}\right)_{i}, \boldsymbol{\phi}_{\mathbf{1 , 0}}\right)\right], N\left(\left(\boldsymbol{\nu}_{\boldsymbol{i}}\right)_{i}, \boldsymbol{\phi}_{\mathbf{1}, \mathbf{0}}, s_{0}\right) \leq 1$, it follows that, for all $i=1, \ldots, k$, we have $\left|\phi_{i}(s)\right| \leq C$, hence from the change of variables (35) and (41) together with the definition (11) of $\bar{\zeta}_{i}(s)$, we see that, for all $i=1, \ldots, k$, we have

$$
\left|\xi_{i}(s)\right|=\frac{2}{p-1}\left|\zeta_{i}(s)-\bar{\zeta}_{i}(s)-\zeta_{0}\right| \leq C
$$

so that for all $i=2, \ldots, k$, we obtain

$$
\left|\zeta_{i}(s)-\zeta_{i-1}(s)-\frac{p-1}{2} \log s\right| \leq C .
$$

This in turns implies that $1 /\left(C s^{\left|\gamma_{i}\right|}\right) \leq 1-d_{i}^{2} \leq C / s^{\left|\gamma_{i}\right|}$, except for $i=(k+1) / 2$ if $k$ is odd, where $1-d_{i}(s)^{2} \geq \frac{1}{C}$. This leads also to the bounds

$$
J \leq \frac{C}{s}, \quad \bar{J} \leq \frac{C}{s^{1 / 2}}, \quad \sum_{j=1}^{k} \phi_{j}^{2} \leq \frac{C}{s^{2 \eta}},
$$

where the different quantities are defined in (30) and (411).

Hence, the estimates (33), (48), (31) and (42) read as follows: for all $s \in\left[s_{0}, s^{*}\left(s_{0},\left(\boldsymbol{\nu}_{\boldsymbol{i}}\right)_{i}, \boldsymbol{\phi}_{\mathbf{1}, \mathbf{0}}\right)\right]$

$$
\begin{aligned}
\|q(s)\|_{\mathscr{H}} & \leq \frac{C}{s^{\bar{p} / 2}}+\frac{C}{s^{\alpha / 2}} \leq \frac{1}{2 s^{1 / 2+\eta}}, \text { and from this } \\
\left|\dot{\nu}_{i}-\nu_{i}\right| & \leq C\left(\frac{1}{s^{\left|\gamma_{i}\right|+\bar{p}}}+\frac{1}{s^{\left|\gamma_{i}\right|+\alpha}}+\frac{1}{s^{\left|\gamma_{i}\right|+1}}\right) \leq \frac{C}{s^{\left|\gamma_{i}\right|+1}} \\
\left|\dot{\phi}_{i}+\frac{m_{i}}{s} \phi_{i}\right| & \leq C\left(\frac{1}{s^{1+2 \eta}}+\frac{1}{s^{3 / 2}}+\frac{1}{s^{(\bar{p}+1) / 2}}+\frac{1}{s^{1+\delta}}+\frac{1}{s^{\alpha}}\right) \leq \frac{C}{s^{1+2 \eta}},
\end{aligned}
$$

provided that $s_{0}$ is large enough (note that we used the definition (45) of $\eta$ in the first and last line above). Now, if $i=2, \ldots, k$, recall from the definition (45) of $\eta$ that $0<2 \eta<m_{i}$. Considering $g_{i}(s)=s^{m_{i}} \phi_{i}(s)$, we see that $\left|\dot{g}_{i}(s)\right| \leq C s^{m_{i}-(1+2 \eta)}$. Since $\phi_{i}\left(s_{0}\right)=0$ by (50), we write

$$
\left|\phi_{i}(s)\right| \leq \frac{C}{s^{2 \eta}} \leq \frac{1}{2 s^{\eta}}
$$

for $s_{0}$ large enough.

Since $N\left(\left(\boldsymbol{\nu}_{\boldsymbol{i}}\right)_{i}, \boldsymbol{\phi}_{\mathbf{1 , 0},}, s^{*}\left(s_{0},\left(\boldsymbol{\nu}_{\boldsymbol{i}}\right)_{i}, \boldsymbol{\phi}_{\mathbf{1}, \mathbf{0}}\right)\right)=1$ by (53) , we see from the definition (51) of $N$ together with (55) and (58) that necessarily there exists $i=1, \ldots, k$ such that

$s^{*}\left(s_{0},\left(\boldsymbol{\nu}_{\boldsymbol{i}}\right)_{i}, \boldsymbol{\phi}_{\mathbf{1}, \mathbf{0}}\right)^{1 / 2+\left|\gamma_{i}\right|}\left|\nu_{i}\left(s^{*}\left(s_{0},\left(\boldsymbol{\nu}_{\boldsymbol{i}}\right)_{i}, \boldsymbol{\phi}_{\mathbf{1}, \mathbf{0}}\right)\right)\right|=1$, or $s^{*}\left(s_{0},\left(\boldsymbol{\nu}_{\boldsymbol{i}}\right)_{i}, \boldsymbol{\phi}_{\mathbf{1}, \mathbf{0}}\right)^{\eta}\left|\phi_{1}\left(s^{*}\left(s_{0},\left(\boldsymbol{\nu}_{\boldsymbol{i}}\right)_{i}, \boldsymbol{\phi}_{\mathbf{1}, \mathbf{0}}\right)\right)\right|=1$.

Using the definitions (54) and (47) of the flow $\Phi$ and the rescaling function $\Gamma_{s}$, we get to the conclusion of part (i). 
(ii) Assume that $\Phi\left(s,\left(\boldsymbol{\nu}_{\boldsymbol{i}}\right)_{i}, \boldsymbol{\phi}_{\mathbf{1}, \mathbf{0}}\right) \in \mathbb{S}$ for some $s \in\left[s_{0}, s^{*}\left(s_{0},\left(\boldsymbol{\nu}_{\boldsymbol{i}}\right)_{i}, \boldsymbol{\phi}_{\mathbf{1 , 0}}\right)\right]$. Therefore, either there exists $i=1, \ldots, k$ such that

$$
s^{1 / 2+\left|\gamma_{i}\right|}\left|\nu_{i}(s)\right|=1,
$$

or

$$
s^{\eta}\left|\phi_{1}(s)\right|=1 .
$$

In the case where (59) holds, by using (56), we write

$$
\begin{gathered}
\frac{d}{d s} s^{1 / 2+\left|\gamma_{i}\right|} \nu_{i}(s)=s^{1 / 2+\left|\gamma_{i}\right|}\left(\left(\frac{1}{2}+\left|\gamma_{i}\right|\right) \frac{\nu_{i}(s)}{s}+\dot{\nu}_{i}(s)\right) \\
=s^{1 / 2+\left|\gamma_{i}\right|}\left(\nu_{i}(s)\left(1+\frac{1}{2 s}+\frac{\left|\gamma_{i}\right|}{s}\right)+O\left(\frac{1}{s^{1+\left|\gamma_{i}\right|}}\right)\right) \\
=s^{1 / 2+\left|\gamma_{i}\right|}\left(\nu_{i}(s)+O\left(\frac{1}{s^{1+\left|\gamma_{i}\right|}}\right)\right) .
\end{gathered}
$$

By (59), we deduce that for $s_{0}$ large enough,

$$
\frac{d}{d s} s^{1 / 2+\left|\gamma_{i}\right|} \nu_{i}(s) \cdot \frac{1}{s^{1 / 2+\left|\gamma_{i}\right|} \nu_{i}(s)} \geq \frac{1}{2} .
$$

Now, in the case where (60) holds, using the fact that $m_{1}=0$ (see (39)), we derive from the inequality (57) the fact that

$$
\left|\frac{d}{d s} \phi_{1}\left(s^{*}\right)\right| \leq \frac{\eta}{2\left(s^{*}\right)^{1+\eta}} .
$$

Since $\frac{d}{d s}\left(\frac{1}{s^{\eta}}\right)=-\frac{\eta}{s^{1+\eta}}$, then we have

- $\frac{d}{d s} \phi_{1}\left(s^{*}\right)>\left.\frac{d}{d s} \frac{1}{s^{\eta}}\right|_{s=s^{*}}$, if $\phi_{1}\left(s^{*}\right)=\frac{1}{s^{* \eta}}$,

- $\frac{d}{d s} \phi_{1}\left(s^{*}\right)<\left.\frac{d}{d s} \frac{-1}{s^{\eta}}\right|_{s=s^{*}}$, if $\phi_{1}\left(s^{*}\right)=-\frac{1}{s^{* \eta}}$,

Thus, the flow is transverse on $\mathbb{B}$ and part (ii) holds. Note that the transversality of $\phi_{1}$ is a new feature in our approach, which was not present in Côte and Zaag [12].

(iii) Let $\left(\left(\boldsymbol{\nu}_{\boldsymbol{i}}\right)_{i}, \boldsymbol{\phi}_{\mathbf{1}, \mathbf{0}}\right) \in \mathbb{S}$. From (48) and the definition (54) of the flow $\Phi$, we see that

$$
\Phi\left(s_{0},\left(\boldsymbol{\nu}_{\boldsymbol{i}}\right)_{i}, \boldsymbol{\phi}_{\mathbf{1 , 0}}\right)=\left(\left(\boldsymbol{\nu}_{\boldsymbol{i}}\right)_{i}, \boldsymbol{\phi}_{\mathbf{1 , 0}}\right) .
$$

Since $\left(\left(\boldsymbol{\nu}_{\boldsymbol{i}}\right)_{i}, \boldsymbol{\phi}_{\mathbf{1}, \mathbf{0}}\right) \in \mathbb{S}$, we can use (ii) of Claim 2.7 and see that the flow $\Phi$ is transverse to $\mathbb{B}$ at $s=s_{0}$. By definition of the exit time, we see that

$$
s^{*}\left(s_{0},\left(\boldsymbol{\nu}_{\boldsymbol{i}}\right)_{i}, \boldsymbol{\phi}_{\mathbf{1 , 0}}\right)=s_{0} .
$$

Using (61), we get to the conclusion of part (iii). This concludes the proof of Claim 2.7 .

We now conclude the proof of Proposition 2.6. From part (ii) of Claim 2.7, $\left(\left(\boldsymbol{\nu}_{\boldsymbol{i}}\right)_{i}, \boldsymbol{\phi}_{\mathbf{1 , 0}}\right) \rightarrow$ $s^{*}\left(s_{0},\left(\boldsymbol{\nu}_{\boldsymbol{i}}\right)_{i}, \boldsymbol{\phi}_{\mathbf{1}, \mathbf{0}}\right)$ is continuous, hence from (i) and (iii),

$$
\left(\left(\boldsymbol{\nu}_{\boldsymbol{i}}\right)_{i}, \boldsymbol{\phi}_{\mathbf{1}, \mathbf{0}}\right) \mapsto \Phi\left(s^{*}\left(s_{0},\left(\boldsymbol{\nu}_{\boldsymbol{i}}\right)_{i}, \boldsymbol{\phi}_{\mathbf{1 , 0}}\right),\left(\boldsymbol{\nu}_{\boldsymbol{i}}\right)_{i}, \boldsymbol{\phi}_{\mathbf{1 , 0}}\right)
$$

is a continuous map from $\mathbb{B}$ to $\mathbb{S}$ whose restriction to $\mathbb{S}$ is the identity. By the index theory, this is a contradiction. Thus, there exists $\left(\left(\boldsymbol{\nu}_{\boldsymbol{i}}\right)_{i}, \boldsymbol{\phi}_{\mathbf{1}, \mathbf{0}}\right) \in \mathbb{B}$ such that for all $s \geq s_{0}, N\left(s_{0},\left(\left(\boldsymbol{\nu}_{\boldsymbol{i}}\right)_{i}, \boldsymbol{\phi}_{\mathbf{1}, \mathbf{0}}\right) \leq 1\right.$, hence $w\left(s_{0},\left(\left(\boldsymbol{\nu}_{\boldsymbol{i}}\right)_{i}, \boldsymbol{\phi}_{\mathbf{0}, \mathbf{1}}, \cdot, s\right) \in \mathscr{V}(s)\right.$. This is the desired conclusion. This concludes the proof of Proposition 2.6. 
It remains to give the proof of Proposition 2.1 in order to conclude this section. Let us first recall from Lemma A.2 in [52] the following continuity result for the family of solitons $\kappa^{*}(d, \nu)$ :

Lemma 2.8 (Continuity of $\left.\kappa^{*}\right)$. For all $A \geq 2$, there exists $C(A)>0$ such that if $\left(d_{1}, \nu_{1}\right)$ and $\left(d_{2}, \nu_{2}\right)$ satisfy

$$
\frac{\nu_{1}}{1-\left|d_{1}\right|}, \frac{\nu_{2}}{1-\left|d_{2}\right|} \in\left[-1+\frac{1}{A}, A\right]
$$

then

$$
\begin{aligned}
& \left\|\kappa^{*}\left(d_{1}, \nu_{1}\right)-\kappa^{*}\left(d_{2}, \nu_{2}\right)\right\|_{\mathscr{H}} \\
& \quad \leq C(A)\left(\left|\frac{\nu_{1}}{1-\left|d_{1}\right|}-\frac{\nu_{2}}{1-\left|d_{2}\right|}\right|+\left|\arg \tanh d_{1}-\arg \tanh d_{2}\right|\right) .
\end{aligned}
$$

With this lemma, we can give the proof of Proposition 2.1.

Proof of Proposition [2.1. Let us consider the solution constructed in Proposition 2.6. Since $w(s) \in \mathscr{V}(s)$ for all $s \geq s_{0}$, from Corollary 2.4 and the definition 2.5 of $\mathscr{V}(s)$, we see that (57) holds. In particular, for $i=1, \ldots, k$, we see that

$$
\left|\phi_{i}(s)\right| \leq \frac{C}{s^{\eta}}, \quad \text { for } \quad s \quad \text { large enough. }
$$

Therefore, for $i=1, \ldots, k$, we have $\phi_{i}(s) \rightarrow 0$ as $s \rightarrow \infty$. Then, by (41), we see that $\xi_{i}(s) \rightarrow 0$, for $i=1, \ldots, k$. From (35) , we see that $\zeta_{i}(s)-\bar{\zeta}_{i}(s)-\zeta_{0} \rightarrow 0$ for all $i=1, \ldots, k$ and (27) follows. In particular,

$$
1-\left|d_{i}(s)\right| \sim C_{i} s^{-\left|\gamma_{i}\right|} \text { as } s \rightarrow \infty,
$$

hence, from the definition 2.5 of $\mathscr{V}(s)$, we have

$$
\forall s \geq s_{0}, \frac{\left|\nu_{i}\right|}{1-\left|d_{i}(s)\right|} \leq C\left(s_{0}\right) s^{-\frac{1}{2}} .
$$

Therefore, Lemma 2.8 applies and since $\kappa^{*}\left(d_{i}(s), 0, y\right)=\left(\kappa\left(d_{i}(s), y\right), 0\right)$, we have

$$
\left\|\kappa^{*}\left(d_{i}(s), \nu_{i}(s)\right)-\left(\kappa\left(d_{i}(s), 0\right)\right)\right\|_{\mathscr{H}} \leq C\left(s_{0}\right) \frac{\left|\nu_{i}\right|}{1-\left|d_{i}(s)\right|} \leq C\left(s_{0}\right) s^{-\frac{1}{2}} .
$$

As $\|q(s)\|_{\mathscr{H}} \leq \frac{C}{s^{\frac{1}{2}+\eta}}$ by definition 2.5 of $\mathscr{V}(s)$, and with (28), we deduce that

$$
\left\|\left(\begin{array}{c}
w(s) \\
\partial_{s} w(s)
\end{array}\right)-\left(\begin{array}{c}
k \\
\sum_{i=1}^{k}(-1)^{i+1} \kappa\left(d_{i}(s)\right) \\
0
\end{array}\right)\right\|_{\mathscr{H}} \leq\|q(s)\|_{\mathscr{H}}+C\left(s_{0}\right) s^{-\frac{1}{2}} \leq C\left(s_{0}\right) s^{-\frac{1}{2}}
$$

and (26) follows. This concludes the proof of Proposition 2.1.

Now, we are ready to give the proof of Theorem 1 (as we said at the beginning of the section, we don't prove Corollary 2 as it follows directly from Theorem 1). 


\section{Proof of Theorem 1}

The proof is very easy, since we have only to translate the construction of the previous section into the $u(x, t)$ setting, and recover a solution to our problem. This part contains straightforward and obvious arguments which may be skipped by specialists. We give them for the reader's convenience.

Consider an integer $k \geq 2$ and consider $w(y, s)$ the solution of (6) constructed in Proposition 2.1.

Then, let us define $u(x, t)$ as the solution of equation (11) with initial data in $\mathrm{H}_{\mathrm{loc}, \mathrm{u}}^{1} \times$ $\mathrm{L}_{\text {loc, } \mathrm{u}}^{2}(\mathbb{R})$ whose trace in $(-1,1)$ is given by

$$
u(x, 0)=w\left(x, s_{0}\right) \text { and } \partial_{t} u(x, 0)=\partial_{s} w\left(x, s_{0}\right)+\frac{2}{p-1} w\left(x, s_{0}\right)+x \partial_{y} w\left(x, s_{0}\right) .
$$

Then $u(x, t)$ satisfies all the requirements in Theorem 1. Indeed, by the finite speed of propagation, we clearly have:

(i) For all $t \in[0,1)$ and $|x|<1-t$,

$$
u(x, t)=(1-t)^{-\frac{2}{p-1}} w\left(\frac{x}{1-t}, s_{0}-\log (1-t)\right) .
$$

Indeed, by definition (5) of similarity variables, the function on the right-hand side of (65) is a solution to equation (10) with the same initial data (64) as $u(x, t)$. Since that initial data is in $H^{1} \times L^{2}(-1,1)$ and equation (11) is well-posed in $H^{1} \times L^{2}$ of sections of backward light cones, both solutions are equal from the uniqueness to the Cauchy problem and the finite speed of propagation, hence (65) holds. In particular, from (5), we have

$$
\forall s \geq 0, \quad \forall y \in(-1,1), \quad w_{0,1}(y, s)=w\left(y, s+s_{0}\right) .
$$

(ii) $u$ is a blow-up solution. Indeed, if not, then $u$ is global and $u \in L_{l o c}^{\infty}\left([0, \infty), \mathrm{H}_{\text {loc, } \mathrm{u}}^{1} \times\right.$ $\left.\mathrm{L}_{\text {loc,u }}^{2}(\mathbb{R})\right)$. In particular, we write from the Sobolev injection, for all $s \geq 0$ and $\epsilon>0$,

$$
\left\|w_{0,1}(s)\right\|_{L_{\rho}^{2}} \leq C\|u\|_{L^{\infty}(|x|<1+\epsilon-t)} e^{-\frac{2 s}{p-1}} \rightarrow 0 \text { as } s \rightarrow \infty .
$$

This is in contradiction with (266) and (26) .

(iii) $T(0)=1$. Indeed, from (66) we see that $u(x, t)$ is defined in the cone $|x|<1-t$, $t \geq 0$, hence $T(0) \geq 1$. From (67), we see that if $T(0)>1+\epsilon$ for some $\epsilon>0$, then the same contradiction follows. Thus $T(0)=1$.

(iv) From above, we can use the simplified notation for (5) and write $w_{0}$ instead of $w_{0,1}$, and rewrite (65) as follows:

$$
\forall s \geq 0, \quad \forall y \in(-1,1), \quad w_{0}(y, s)=w\left(y, s+s_{0}\right) .
$$

Using (26) and (27), we see that (15) follows for $w_{0}$ with

$$
\zeta_{i}(s)-\bar{\zeta}_{i}(s) \rightarrow \zeta_{0} \text { as } s \rightarrow \infty \text { for } i=1, \ldots, k
$$


where $\zeta_{0} \in \mathbb{R}$ and $\left(\bar{\zeta}_{i}(s)\right)_{i}$ is the explicit solution of system (12). Thanks to the following continuity result for the solitons $\kappa(d)$ (which follows from estimate (63) in Lemma 2.8):

$$
\left\|\kappa\left(d_{1}\right)-\kappa\left(d_{2}\right)\right\|_{\mathscr{H}_{0}} \leq C\left|\arg \tanh d_{1}-\arg \tanh d_{2}\right|,
$$

we see that (15) still holds if we slightly modify the $\zeta_{i}(s)$ by putting $\zeta_{i}(s)=\bar{\zeta}_{i}(s)+\zeta_{0}$ as required by (16). Finally, if we consider the more restrictive hypothesis $\left(H^{\prime}\right)$, from the classification of the blow-up behavior for general solutions, available from [30], we clearly see that the origin is a characteristic point. Thus, we have a solution obeying all the requirements of Theorem 1 .

\section{A Dynamics of equation (6) near multi-solitons}

This appendix is devoted to the proof of Proposition 2.3. In the special case where $(f, g) \equiv(0,0)$, the proof is already known from [12, Proposition 3.4]. Note that this proof is based on Lemma C.2, Claims 4.8 and 4.9 of [52], together with Proposition 3.2 in [51].

In our case, where $(f, g) \not \equiv(0,0)$, in order to avoid unnecessary repetition, we kindly refer the reader to [12] and [52] for all the projections of the terms in (69) not involving $f$ and $g$ and we will only focus here on the terms with $f$ and $g$.

Proof of Proposition [2.3. Using the definition (28) of $q$, we transform equation (6) satisfied by $w$ into the following system satisfied by $q$, for all $s \in\left[s_{0}, \bar{s}\right)$ :

$$
\begin{aligned}
\frac{\partial}{\partial s}\left(\begin{array}{l}
q_{1} \\
q_{2}
\end{array}\right) & =\hat{L}\left(\begin{array}{l}
q_{1} \\
q_{2}
\end{array}\right)-\sum_{j=1}^{k}(-1)^{j}\left[\left(\nu_{j}^{\prime}(s)-\nu_{j}(s)\right) \partial_{\nu} \kappa^{*}+d_{j}^{\prime}(s) \partial_{d} \kappa^{*}\right]\left(d_{j}(s), \nu_{j}(s), y\right) \\
& +\left(\begin{array}{c}
0 \\
R
\end{array}\right)+\left(\begin{array}{c}
0 \\
h\left(q_{1}\right)
\end{array}\right)+\left(\begin{array}{c}
0 \\
\tilde{f}\left(q_{1}\right)
\end{array}\right)+\left(\begin{array}{c}
0 \\
\tilde{g}\left(q_{1}\right)
\end{array}\right),
\end{aligned}
$$

where

$$
\begin{aligned}
\hat{L}\left(\begin{array}{l}
q_{1} \\
q_{2}
\end{array}\right) & =\left(\begin{array}{c}
q_{2} \\
\mathscr{L} q_{1}+\psi q_{1}-\frac{p+3}{p-1} q_{2}-2 y \partial_{y} q_{2}
\end{array}\right) \\
\psi(y, s) & =p\left|K_{1}^{*}(y, s)\right|^{p-1}-\frac{2(p+1)}{(p-1)^{2}}, \quad K_{1}^{*}(y, s)=\sum_{j=1}^{k}(-1)^{j} \kappa_{1}^{*}\left(d_{j}(s), \nu_{j}(s), y\right) \\
h\left(q_{1}\right) & =\left|K_{1}^{*}+q_{1}\right|^{p-1}\left(K_{1}^{*}+q_{1}\right)-\left|K_{1}^{*}\right|^{p-1} K_{1}^{*}-p\left|K_{1}^{*}\right|^{p-1} q_{1}, \\
\tilde{f}\left(q_{1}\right) & =e^{-\frac{2 p s}{p-1}} f\left(e^{\frac{2 s}{p-1}}\left(K_{1}^{*}+q_{1}\right)\right) \\
\tilde{g}\left(q_{1}\right) & =e^{-\frac{2 p s}{p-1}} g\left(x_{0}+y e^{-s}, T_{0}-e^{-s}, e^{\frac{2 s}{p-1}} w, e^{\frac{(p+1) s}{p-1}} \partial_{y} w, e^{\frac{(p+1) s}{p-1}}\left(\partial_{s} w+y \partial_{y} w+\frac{2 w}{p-1}\right)\right) \\
R & =\left|K_{1}^{*}\right|^{p-1} K_{1}^{*}-\sum_{j=1}^{k}(-1)^{j} \kappa_{1}^{*}\left(d_{j}(s), \nu_{j}(s), y\right)^{p},
\end{aligned}
$$

As in the unperturbed case, we give a decomposition of the solution which is well adapted to the proof. More precisely, we start by localizing equation (69) near the center 
$d_{i}^{*}(s)=\frac{d_{i}(s)}{1+\nu_{i}(s)}$ of $\kappa^{*}\left(d_{i}(s), \nu_{i}(s)\right)$ for each $i=1, \ldots, k$, which allows us to view it locally as a perturbation of the case of $\kappa(d, y)$ already treated in [47]. For this, given $i=1, \ldots, k$, we need to expand the linear operator of equation (69) as follows:

$$
\hat{L}(q)=\hat{L}_{d_{i}^{*}(s)}(q)+\left(0, \bar{V}_{i}(y, s) q_{1}\right)+\left(0, V_{i}^{*}(y, s) q_{1}\right),
$$

with

$$
\begin{aligned}
\hat{L}_{d}\left(\begin{array}{l}
q_{1} \\
q_{2}
\end{array}\right) & =\left(\begin{array}{c}
q_{2} \\
\mathscr{L} q_{1}+\psi^{*}(d) q_{1}-\frac{p+3}{p-1} q_{2}-2 y q_{2}^{\prime}
\end{array}\right), \\
\psi^{*}(d, y) & =p \kappa(d, y)^{p-1}-\frac{2(p+1)}{(p-1)^{2}} \\
\bar{V}_{i}(y, s) & =p \kappa_{1}^{*}\left(d_{i}(s), \nu_{i}(s), y\right)^{p-1}-p \kappa\left(d_{i}^{*}(s), y\right)^{p-1}, \\
V_{i}^{*}(y, s) & =p\left|K_{1}^{*}(y, s)\right|^{p-1}-p \kappa_{1}^{*}\left(d_{i}(s), \nu_{i}(s), y\right)^{p-1} .
\end{aligned}
$$

We proceed in two parts.

- In Part 1, in order to prove (31) and (32), we project equation (69) using the projector $\Pi_{l}^{d_{i}^{*}(s)}$ defined in (20) with $l=0,1$ and $d_{i}^{*}(s)=\frac{d_{i}(s)}{1+\nu_{i}(s)}$.

- In Part 2, we will find a Lyapunov functional for equation (69), which is equivalent to the norm squared, and we thus obtain estimate (33).

\section{Part 1: Projection of equation (69) on $F_{0}^{d_{i}^{*}(s)}$ and $F_{1}^{d_{i}^{*}(s)}$}

Let us assume that $s_{0} \geq 1$ is large enough and take $s \in\left[s_{0}, \bar{s}\right)$.

The proof of (31) and (32) is similar to the proof in [12] except for the treatment of the perturbation terms. More precisely, let $i=1, \ldots, k$ be fixed and $l=0$ or 1 , the projector $\Pi_{l}^{d_{i}^{*}(s)}$ defined in (20) is now applied for each term of equation (69). Thanks to (29) together with the analysis of [51, Appendix C], [52, Appendix C] and [12, Appendix A], we easily obtain the following estimates related to the terms not involving $f$ and $g$ :

$$
\begin{aligned}
& \left|\Pi_{l}^{d_{i}^{*}}\left(\partial_{s} q\right)\right| \leq C \frac{\left|d_{i}^{\prime}\right|+\left|\nu_{i}^{\prime}-\nu_{i}\right|+\left|\nu_{i}\right|}{1-d_{i}^{* 2}}\|q\|_{\mathscr{H}}, \\
& \Pi_{l}^{d_{i}^{*}}\left(\hat{L}_{d_{i}^{*}}(q)\right)=l \Pi_{l}^{d_{i}^{*}}(q)=0, \\
& \left|\Pi_{l}^{d_{i}^{*}}\left(0, h\left(q_{1}\right)\right)\right| \leq C\|q\|_{\mathscr{H}}^{2}, \\
& \left|\Pi_{l}^{d_{i}^{*}}\left(0, V_{i}^{*} q_{1}\right)\right| \leq C\|q\|_{\mathscr{H}}^{2}+C\left(J^{*}\right)^{1+\delta_{1}}, \\
& \left|\Pi_{l}^{d_{i}^{*}}\left(0, \bar{V}_{i} q_{1}\right)\right| \leq C \frac{\left|\nu_{i}\right|}{1-d_{i}^{* 2}}\|q\|_{\mathscr{H}}, \\
& \left|\Pi_{l}^{d_{i}^{*}}(0, R)\right| \leq C J^{*} \\
& \left|\Pi_{0}^{d_{i}^{*}}(0, R)-c_{2}(p)(-1)^{i}\left[e^{-\frac{2}{p-1}\left(\zeta_{i}-\zeta_{i-1}\right)}-e^{-\frac{2}{p-1}\left(\zeta_{i+1}-\zeta_{i}\right)}\right]\right| \leq C J^{1+\delta_{2}}+C J \bar{J},
\end{aligned}
$$

where $\delta_{1}>0, \delta_{2}>0$ and $c_{2}(p)>0$, where

$$
J^{*}=\sum_{i=1}^{k-1} e^{-\frac{2}{p-1}\left(\zeta_{i+1}^{*}-\zeta_{i}^{*}\right)},
$$


and $\zeta_{i}^{*}=-\operatorname{argth}\left(d_{i}^{*}\right)$. Moreover, we recall some estimates related to the projections of $\partial_{\nu} \kappa^{*}\left(d_{i}, \nu_{i}\right)$ and $\partial_{d} \kappa^{*}\left(d_{i}, \nu_{i}\right)$ given in [52, Claim 3.2] and in [12, Appendix A]

$$
\begin{aligned}
& \Pi_{0}^{d_{i}^{*}}\left(\partial_{\nu} \kappa^{*}\left(d_{i}, \nu_{i}\right)\right)=0 \\
& -\frac{C}{1-d_{i}^{* 2}} \leq \Pi_{1}^{d_{i}^{*}}\left(\partial_{\nu} \kappa^{*}\left(d_{i}, \nu_{i}\right)\right) \leq-\frac{1}{C\left(1-d_{i}^{* 2}\right)}, \\
& \left|\Pi_{1}^{d_{i}^{*}}\left(\partial_{d} \kappa^{*}\left(d_{i}, \nu_{i}\right)\right)\right| \leq \frac{C}{1-d_{i}^{* 2}}, \\
& -\frac{C}{1-d_{i}^{* 2}} \leq \Pi_{0}^{d_{i}^{*}}\left(\partial_{d} \kappa^{*}\left(d_{i}, \nu_{i}\right)\right) \leq-\frac{1}{C\left(1-d_{i}^{* 2}\right)}, \\
& \left|\Pi_{l}^{d_{i}^{*}}\left(\partial_{\nu} \kappa^{*}\left(d_{j}, \nu_{j}\right)\right)\right|+\left|\Pi_{l}^{d_{i}^{*}}\left(\partial_{d} \kappa^{*}\left(d_{j}, \nu_{j}\right)\right)\right| \leq \frac{C}{1-d_{j}^{* 2}} \sqrt{J^{*}}, \quad \text { for } \quad i \neq j, \\
& \left|\Pi_{0}^{d_{i}^{*}}\left(\partial_{d} \kappa^{*}\left(d_{i}, \nu_{i}\right)\right)+\frac{c_{3}}{1-d_{i}^{2}}\right| \leq C \bar{J},
\end{aligned}
$$

for some $c_{3}>0$.

In the sequel, we shall intensively use the following inequalities

$$
C^{-1} J \leq J^{*} \leq C J, \quad C^{-1} \leq \frac{1-d_{i}^{2}}{1-d_{i}^{* 2}} \leq C,
$$

and

$$
C^{-1}\left(J^{*}\right)^{\frac{\bar{p}}{2}} \leq \hat{J}^{*} \leq C\left(J^{*}\right)^{\frac{\bar{p}}{2}}
$$

where $J$ and $J^{*}$ are defined in (30) and (82) and

$$
\hat{J}^{*}=\sum_{i=1}^{k-1} e^{-\frac{\bar{p}}{p-1}\left(\zeta_{i+1}^{*}-\zeta_{i}^{*}\right)} .
$$

As for the proof of (84) and (85), note that (84) easily follows from (29), and that (85) follows from the fact that all the norms on $\mathbb{R}^{k-1}$ are equivalent.

Now, we focus on the "new" terms of the problem related to the perturbation terms $f$ and $g$.

Let us first recall from our previous papers the following basic bounds on the solitons $\kappa(d, y)$ defined in (10) and $\kappa^{*}(d, \nu, y)$ defined in (18) together with a crucial Hardy Sobolev inequality:

\section{Lemma A.1. (Useful estimates)}

(i)(A Hardy-Sobolev type inequality) For all $h \in \mathscr{H}_{0}$, we have

$$
\|h\|_{L_{\frac{\rho}{1-y^{2}}(-1,1)}}+\|h\|_{L_{\rho}^{p+1}(-1,1)}+\left\|h\left(1-y^{2}\right)^{\frac{1}{p-1}}\right\|_{L^{\infty}(-1,1)} \leq C\|h\|_{\mathscr{H}_{0}} .
$$

(ii) (Boundedness of $\kappa(d, y)$ in several norms) For all $d \in(-1,1)$, we have $\|\kappa(d, y)\|_{L_{\frac{\rho}{1-y^{2}}(-1,1)}}+\|\kappa(d, y)\|_{L_{\rho}^{p+1}(-1,1)}+\left\|\kappa(d, y)\left(1-y^{2}\right)^{\frac{1}{p-1}}\right\|_{L^{\infty}(-1,1)}+\|\kappa(d, y)\|_{\mathscr{H}_{0}} \leq C$. 
(iii) For $l=0,1$ and any $|y|<1,\left|W_{l, 2}(d, y)\right| \leq C \kappa(d, y) \leq C\left(1-y^{2}\right)^{-\frac{1}{p-1}}$.

(iv) (Properties of $\left.\kappa^{*}(d, \nu, y)\right)$ For all $d \in(-1,1)$ and $\nu>-1+|d|$, we have:

$$
\begin{aligned}
\forall y \in(-1,1), 0 \leq \kappa_{1}^{*}(d, \nu, y)=\lambda \kappa\left(\frac{d}{1+\nu}, y\right) & \leq \frac{C \lambda}{\left(1-y^{2}\right)^{\frac{1}{p-1}}}, \\
\left\|\kappa^{*}(d, \nu)\right\|_{\mathscr{H}} & \leq C \lambda+C 1_{\{\nu<0\}} \frac{|\nu|}{\sqrt{1-d^{2}}} \lambda^{\frac{p+1}{2}},
\end{aligned}
$$

where $\lambda$ is defined by

$$
\lambda=\lambda(d, \nu)=\frac{\left(1-d^{2}\right)^{\frac{1}{p-1}}}{\left[(1+\nu)^{2}-d^{2}\right]^{\frac{1}{p-1}}} .
$$

(v) For all $d \in(-1,1)$ and $\nu>-1+|d|$, we have:

$$
\forall y \in(-1,1), \quad\left|\partial_{d} \kappa_{1}^{*}(d, \nu, y)\right|+\left|\partial_{\nu} \kappa_{1}^{*}(d, \nu, y)\right| \leq C \frac{\kappa\left(d^{*}, y\right)}{1-d^{* 2}}, \text { where } d^{*}=\frac{d}{1+\nu} .
$$

Proof. - For (i), see Lemma 2.2 page 51 in [47. For (ii), use (i) and identity (49) page 59 in [47]. For (iii), see (196) page 105 in [47]. For (iv), see (i) in Lemma A.2 page 2878 in [52. For (v), see (3.41) page 2857 in [52].

In the following, we give the estimates involving the perturbation terms.

- Estimate of $\Pi_{l}^{d_{i}^{*}}\left(0, \tilde{f}\left(q_{1}\right)\right)$, for $l=0,1$.

Using the definition (20) and (iii) in Lemma A.1, we conclude that

$$
\left|\Pi_{l}^{d_{i}^{*}}\left(0, \tilde{f}\left(q_{1}\right)\right)\right| \leq C \int_{-1}^{1}\left|\tilde{f}\left(q_{1}\right)\right| \kappa\left(d_{i}^{*}\right) \rho d y .
$$

By using the definition (73) of $\tilde{f}$, the condition $(H)$ and (iii) in Lemma A.1, we deduce that

$$
\left|\Pi_{l}^{d_{i}^{*}}\left(0, \tilde{f}\left(q_{1}\right)\right)\right| \leq C e^{-\frac{2 p s}{p-1}}+C \int_{-1}^{1} \frac{\left|K_{1}^{*}+q_{1}\right|^{p}}{\left(\log \left(2+e^{\frac{4 s}{p-1}}\left(K_{1}^{*}+q_{1}\right)^{2}\right)\right)^{\alpha}} \kappa\left(d_{i}^{*}\right) \rho d y .
$$

In order to bound this latter integral, we divide the interval $[-1,1]$ in two parts:

$A_{1}(s)=\left\{y \in(-1,1)|| K_{1}^{*}+q_{1} \mid \leq e^{-\frac{s}{p-1}}\right\}$ and $A_{2}(s)=\left\{y \in(-1,1)|| K_{1}^{*}+q_{1} \mid>e^{-\frac{s}{p-1}}\right\}$.

On the one hand, if $y \in A_{1}(s)$, we have

$$
\frac{\left|K_{1}^{*}+q_{1}\right|^{p}}{\left(\log \left(2+e^{\frac{4 s}{p-1}}\left(K_{1}^{*}+q_{1}\right)^{2}\right)\right)^{\alpha}} \leq C e^{-\frac{p s}{p-1}} .
$$

Integrating (89) over $A_{1}(s)$ and using (iii) in Lemma A.1, we see that

$$
\int_{A_{1}(s)} \frac{\left|K_{1}^{*}+q_{1}\right|^{p}}{\left(\log \left(2+e^{\frac{4 s}{p-1}}\left(K_{1}^{*}+q_{1}\right)^{2}\right)\right)^{\alpha}} \kappa\left(d_{i}^{*}\right) \rho d y \leq C e^{-\frac{p s}{p-1}} .
$$


On the other hand, if $y \in A_{2}(s)$, we have

$$
\log \left(2+e^{\frac{4 s}{p-1}}\left(K_{1}^{*}+q_{1}\right)^{2}\right)>\log \left(2+e^{\frac{2 s}{p-1}}\right) \geq \frac{2 s}{p-1},
$$

and we write, for all $y \in A_{2}(s)$,

$$
\frac{\left|K_{1}^{*}+q_{1}\right|^{p}}{\left(\log \left(2+e^{\frac{4 s}{p-1}}\left(K_{1}^{*}+q_{1}\right)^{2}\right)\right)^{\alpha}} \leq \frac{C}{s^{\alpha}}\left|K_{1}^{*}+q_{1}\right|^{p} \leq \frac{C}{s^{\alpha}}\left(\left|K_{1}^{*}\right|^{p}+\left|q_{1}\right|^{p}\right) .
$$

If we integrate (91) over $A_{2}(s)$, using the simple fact that $A_{2}(s) \subset[-1,1]$, we obtain

$$
\int_{A_{2}(s)\left(\log \left(2+e^{\frac{4 s}{p-1}}\left(K_{1}^{*}+q_{1}\right)^{2}\right)\right)^{\alpha}} \kappa\left(d_{i}^{*}\right) \rho d y \leq \frac{C}{s^{\alpha}} \int_{-1}^{1}\left(\left|K_{1}^{*}\right|^{p}+\left|q_{1}\right|^{p}\right) \kappa\left(d_{i}^{*}\right) \rho d y .
$$

Furthermore, we apply the inequality $a b^{p} \leq C\left(a^{p+1}+b^{p+1}\right)$, for all $a>0, b>0$, to get

$$
\int_{-1}^{1}\left|q_{1}\right|^{p} \kappa\left(d_{i}^{*}\right) \rho d y \leq C \int_{-1}^{1}\left|q_{1}\right|^{p+1} \rho d y+C \int_{-1}^{1} \kappa^{p+1}\left(d_{i}^{*}\right) \rho d y .
$$

Gathering (92), (93), Lemma A.1 and (29), we have

$$
\int_{-1}^{1}\left|q_{1}\right|^{p} \kappa\left(d_{i}^{*}\right) \rho d y \leq C\|q\|_{\mathscr{H}}^{p+1}+C \leq C .
$$

It suffices to combine (88), (90), (92), (94) and (iv) in Lemma A.1, to obtain that

$$
\left|\Pi_{l}^{d_{i}^{*}}\left(0, \tilde{f}\left(q_{1}\right)\right)\right| \leq \frac{C}{s^{\alpha}} .
$$

- Estimate of $\Pi_{l}^{d_{i}^{*}}\left(0, \tilde{g}\left(q_{1}\right)\right)$, for $l=0,1$.

Proceeding as for estimate (87), we have

$$
\left|\Pi_{l}^{d_{i}^{*}}\left(0, \tilde{g}\left(q_{1}\right)\right)\right| \leq C \int_{-1}^{1}\left|\tilde{g}\left(q_{1}\right)\right| \kappa\left(d_{i}^{*}\right) \rho d y .
$$

By using the condition $(H)$ and the definition (74) of $\tilde{g}$, we obtain

$$
\begin{aligned}
\left|\tilde{g}\left(q_{1}\right)\right| \leq & C e^{-s}\left(\left|\partial_{y} K_{1}^{*}+\partial_{y} q_{1}\right|+\left|\partial_{s} K_{1}^{*}+\partial_{s} q_{1}\right|\right) \\
& \left.+C e^{-s}\left|K_{1}^{*}+q_{1}\right|+\left|K_{1}^{*}+q_{1}\right|^{\frac{p+1}{2}}\right)+C e^{-\frac{2 p s}{p-1}},
\end{aligned}
$$

where $K_{1}^{*}$ is defined in (71). Combining (97) and the expression

$$
q_{2}=\partial_{s} q_{1}+\partial_{s} K_{1}^{*}-\sum_{j=1}^{k}(-1)^{j} \nu_{j} \partial_{\nu} \kappa_{1}^{*}\left(d_{j}, \nu_{j}\right)
$$

(which comes from (28) ), one easily obtains

$$
\left|\tilde{g}\left(q_{1}\right)\right| \leq C e^{-s}\left(\left|\partial_{y} q_{1}\right|+\left|q_{2}\right|+\left|q_{1}\right|+\left|q_{1}\right|^{\frac{p+1}{2}}\right)+C e^{-s}\left(\left|\partial_{y} K_{1}^{*}\right|+\left|K_{1}^{*}\right|+\left|K_{1}^{*}\right|^{\frac{p+1}{2}}\right)
$$




$$
+C e^{-s} \sum_{j=1}^{k}\left|\nu_{j}\right|\left|\partial_{\nu} \kappa_{1}^{*}\left(d_{j}, \nu_{j}\right)\right|+C e^{-\frac{2 p s}{p-1}} .
$$

According to (96), (99), together with items (i) and (ii) in Lemma A.1, we deduce that

$$
\begin{aligned}
&\left|\Pi_{l}^{d_{i}^{*}}\left(0, \tilde{g}\left(q_{1}\right)\right)\right| \leq \underbrace{C e^{-s} \int_{-1}^{1}\left(\left|\partial_{y} q_{1}\right|+\left|q_{2}\right|+\left|q_{1}\right|+\left|q_{1}\right|^{\frac{p+1}{2}}\right) \kappa\left(d_{i}^{*}\right) \rho d y}_{I_{1}(s)} \\
&+\underbrace{C e^{-s} \int_{-1}^{1}\left(\left|\partial_{y} K_{1}^{*}\right|+\left|K_{1}^{*}\right|+\left|K_{1}^{*}\right|^{\frac{p+1}{2}}\right) \kappa\left(d_{i}^{*}\right) \rho d y}_{I_{2}(s)} \\
&+\underbrace{C e^{-s} \sum_{j=1}^{k}\left|\nu_{j}\right| \int_{-1}^{1}\left|\partial_{\nu} \kappa_{1}^{*}\left(d_{j}, \nu_{j}\right)\right| \kappa\left(d_{i}^{*}\right) \rho d y}_{I_{3}(s)}+C e^{-\frac{2 p s}{p-1}} .
\end{aligned}
$$

We are going now to estimate the different terms of the right-hand side of inequality (100). Thanks to the the classical inequality $a b \leq a^{2}+b^{2}$, (ii) in Lemma A.1 and (29), we conclude that

$$
\left|I_{1}(s)\right| \leq C e^{-s} \int_{-1}^{1}\left(\left|\partial_{y} q_{1}\right|^{2}\left(1-y^{2}\right)+\left|q_{2}\right|^{2}+\left|q_{1}\right|^{2}+\left|q_{1}\right|^{p+1}+\frac{\kappa^{2}\left(d_{i}^{*}\right)}{1-y^{2}}\right) \rho d y \leq C e^{-s} .
$$

Proceeding as for estimate (101) and exploiting, (29), (i) and (iv) in Lemma A.1. we conclude

$$
\left|I_{2}(s)\right| \leq C e^{-s}
$$

Using (v) in Lemma A.1 and (84), we see that

$$
\left|I_{3}(s)\right| \leq C e^{-s} \sum_{j=1}^{k} \frac{\left|\nu_{j}\right|}{1-d_{j}^{2}} \int_{-1}^{1}\left|\kappa\left(d_{j}^{*}\right)\right| \kappa\left(d_{i}^{*}\right) \rho d y .
$$

Thanks to (103), (29), the classical inequality $a b \leq a^{2}+b^{2}$, (ii) in Lemma A.1, we conclude

$$
\left|I_{3}(s)\right| \leq C e^{-s} .
$$

Combining the inequalities (100), (101), (102), (104), we infer that

$$
\left|\Pi_{l}^{d_{i}^{*}}\left(0, \tilde{g}\left(q_{1}\right)\right)\right| \leq C e^{-s} .
$$

\section{Conclusion of the proof of (31) and (32):}

We first project equation (69) with $\Pi_{0}^{d_{i}^{*}}$. Using (81), (83), (95), (105) and (84), we conclude

$$
\left|(-1)^{i+1} d_{i}^{\prime} \Pi_{0}^{d_{i}^{*}}\left(\partial_{d} \kappa^{*}\right)+\Pi_{0}^{d_{i}^{*}}(0, R)\right| \leq C \sum_{j=1, j \neq i}^{k} \frac{\left|\nu_{j}^{\prime}-\nu_{j}\right|+\left|d_{j}^{\prime}\right|}{1-d_{j}^{2}} \sqrt{J}
$$




$$
+C \frac{\left|d_{i}^{\prime}\right|+\left|\nu_{i}^{\prime}-\nu_{i}\right|+\left|\nu_{i}\right|}{1-d_{i}{ }^{2}}\|q\|_{\mathscr{H}}+C\left\|_{q}\right\|_{\mathscr{H}}^{2}+C J^{1+\delta_{1}}+\frac{C}{s^{\alpha}} .
$$

By using (83) again, (29), (81), the inequalities (106) and (84), we obtain

$$
\begin{array}{r}
\left|\frac{\dot{\zeta}_{i}}{c_{1}}-\left(e^{-\frac{2}{p-1}\left(\zeta_{i}-\zeta_{i-1}\right)}-e^{-\frac{2}{p-1}\left(\zeta_{i+1}-\zeta_{i}\right)}\right)\right| \leq C \sum_{j=1, j \neq i}^{k} \frac{\left|\nu_{j}^{\prime}-\nu_{j}\right|+\left|d_{j}^{\prime}\right|}{1-d_{j}{ }^{2}} \sqrt{J} \\
+C \frac{\left|d_{i}^{\prime}\right|+\left|\nu_{i}^{\prime}-\nu_{i}\right|+\left|\nu_{i}\right|}{1-d_{i}{ }^{2}}\|q\|_{\mathscr{H}}+C\|q\|_{\mathscr{H}}^{2}+C J^{1+\delta_{3}} \\
+C J \bar{J}+C\left|d_{i}^{\prime}\right| \bar{J}+\frac{C}{s^{\alpha}},
\end{array}
$$

for some $\delta_{3}>0$, where $d_{i}=-\tanh \zeta_{i}$ and $J$ and $\bar{J}$ are defined in (30). Recalling the definition (30) of $\zeta_{i}(s)$ and $J$, we write

$$
\begin{aligned}
\frac{\left|d_{i}^{\prime}\right|}{1-d_{i}^{2}} \leq C \sum_{j=1, j \neq i}^{k} \frac{\left|\nu_{j}^{\prime}-\nu_{j}\right|+\left|d_{j}^{\prime}\right|}{1-d_{j}{ }^{2}} \sqrt{J} & +C \frac{\left|d_{i}^{\prime}\right|+\left|\nu_{i}^{\prime}-\nu_{i}\right|+\left|\nu_{i}\right|}{1-d_{i}{ }^{2}}\|q\|_{\mathscr{H}} \\
& +C\|q\|_{\mathscr{H}}^{2}+C J+C\left|d_{i}^{\prime}\right| \bar{J}+\frac{C}{s^{\alpha}}
\end{aligned}
$$

Now, we use the projection $\Pi_{1}^{d_{i}^{*}}$. Proceeding as for estimate (107), we use the projections of $\partial_{\nu} \kappa^{*}\left(d_{j}, \nu_{j}\right)$ and $\partial_{d} \kappa^{*}\left(d_{j}, \nu_{j}\right)$, given in (83) and the inequality (84) to deduce that

$$
\begin{aligned}
\frac{\left|\nu_{i}^{\prime}-\nu_{i}\right|}{1-d_{i}{ }^{2}} & \leq C \frac{\left|d_{i}^{\prime}\right|}{1-d_{i}{ }^{2}}+C \sum_{j=1, j \neq i}^{k} \frac{\left|\nu_{j}^{\prime}-\nu_{j}\right|+\left|d_{j}^{\prime}\right|}{1-d_{j}{ }^{2}} \sqrt{J} \\
& +C \frac{\left|d_{i}^{\prime}\right|+\left|\nu_{i}^{\prime}-\nu_{i}\right|+\left|\nu_{i}\right|}{1-d_{i}{ }^{2}}\|q\|_{\mathscr{H}}+C\|q\|_{\mathscr{H}}^{2}+C J+\frac{C}{s^{\alpha}}
\end{aligned}
$$

hence from (108),

$$
\begin{aligned}
\frac{\left|\nu_{i}^{\prime}-\nu_{i}\right|}{1-d_{i}{ }^{2}} \leq & C \sum_{j=1, j \neq i}^{k} \frac{\left|\nu_{j}^{\prime}-\nu_{j}\right|+\left|d_{j}^{\prime}\right|}{1-d_{j}{ }^{2}} \sqrt{J}+C \frac{\left|d_{i}^{\prime}\right|+\left|\nu_{i}^{\prime}-\nu_{i}\right|+\left|\nu_{i}\right|}{1-d_{i}{ }^{2}}\|q\|_{\mathscr{H}} \\
& +C\|q\|_{\mathscr{H}}^{2}+C J+C\left|d_{i}^{\prime}\right| \bar{J}+\frac{C}{s^{\alpha}} .
\end{aligned}
$$

Summing-up the estimates (108) and (110) in $i$, using the smallness of $\|q\|_{\mathscr{H}}, \bar{J}$ and $J$, for $s_{0}$ large enough (see (29)), we conclude

$$
\frac{\left|\dot{\nu}_{i}-\nu_{i}\right|}{1-d_{i}^{2}}+\frac{\left|d_{i}^{\prime}\right|}{1-d_{i}{ }^{2}} \leq C\left(\|q\|_{\mathscr{H}}^{2}+J+\|q\|_{\mathscr{H}} \bar{J}\right)+\frac{C}{s^{\alpha}} .
$$

Clearly, using (107) and (111), we conclude (31) and (32).

Part 2: A Lyapunov functional for equation (69)

Let us assume that $s_{0} \geq 1$ is large enough and take $s \in\left[s_{0}, \bar{s}\right)$. 
We now prove estimate (33). Like for Claim 4.8 page 2867 in [52, the idea is to construct a Lyapunov functional for equation (69) which is equivalent to the norm squared. Let us introduce for all $s \in\left[s_{0}, \bar{s}\right]$,

$$
\begin{aligned}
& E_{1}(s)=\frac{1}{2} \varphi(q, q)+R_{-}(s) \\
& E_{2}(s)=E_{1}(s)+\eta \int_{-1}^{1} q_{1} q_{2} \rho d y
\end{aligned}
$$

where $\eta \in(0,1]$ will be fixed at the the end of the proof of (33) and $R_{-}, \varphi, \mathscr{H}\left(q_{1}\right)$ and $\mathscr{F}\left(q_{1}\right)$ are given by:

$$
\begin{aligned}
& R_{-}(s)=-\int_{-1}^{1} \mathscr{H}\left(q_{1}\right) \rho d y-e^{-\frac{2(p+1) s}{p-1}} \int_{-1}^{1} \mathscr{F}\left(e^{\frac{2 s}{p-1}}\left(K_{1}^{*}+q_{1}\right)\right) \rho d y \\
& \varphi(r, q)=\int_{-1}^{1}\left(r_{1}^{\prime} q_{1}^{\prime}\left(1-y^{2}\right)-\psi r_{1} q_{1}+r_{2} q_{2}\right) \rho d y \\
& \mathscr{H}\left(q_{1}\right)=\int_{0}^{q_{1}} h(\xi) d \xi=\frac{\left|K_{1}^{*}+q_{1}\right|^{p+1}}{p+1}-\frac{\left|K_{1}^{*}\right|^{p+1}}{p+1}-\left|K_{1}^{*}\right|^{p-1} K_{1}^{*} q_{1}-\frac{p}{2}\left|K_{1}^{*}\right|^{p-1} q_{1}^{2}, \\
& \mathscr{F}\left(q_{1}\right)=\int_{0}^{q_{1}} \tilde{f}(\xi) d \xi
\end{aligned}
$$

where $\psi(d, y), h\left(q_{1}\right), \tilde{f}\left(q_{1}\right)$ and $K_{1}^{*}$ are defined in (71), (772), (73), respectively.

Before starting the proof of (33), let us give in the following lemma some useful estimates.

Lemma A.2. For all $s \in\left[s_{0}, \bar{s}\right)$, we have:

$$
\begin{array}{r}
C^{-1}\|q\|_{\mathscr{H}}^{2} \leq \varphi(q, q) \leq C\|q\|_{\mathscr{H}}^{2}, \\
\left|\int_{-1}^{1} \mathscr{H}\left(q_{1}\right) \rho d y\right| \leq C\|q\|_{\mathscr{H}}^{1+\min (p, 2)} \leq C s_{0}^{(1-\min (p, 2)) / 2}\|q\|_{\mathscr{H}}^{2}, \\
\left|e^{-\frac{2(p+1) s}{p-1}} \int_{-1}^{1} \mathscr{F}\left(e^{\frac{2 s}{p-1}}\left(K_{1}^{*}+q_{1}\right)\right) \rho d y\right| \leq \frac{C}{s^{\alpha}}, \\
\left|\int_{-1}^{1}\left(K_{1}^{*}+q_{1}\right) \tilde{f}\left(q_{1}\right) \rho d y\right| \leq \frac{C}{s^{\alpha}} .
\end{array}
$$

Proof.

- According to (29) and (84), we know that $\sum_{i=1}^{k} \frac{\left|\nu_{i}\right|}{1-\mid d_{i}^{*}}$ and $\|q\|_{\mathscr{H}}$ are small enough, for $s_{0}$ large enough. Then we can adapt with no difficulty the proof given in [52] (page 2898) to deduce the estimates (115) and (116).

- Clearly, using similar arguments to the proof of (95), we prove the following estimate:

$$
\left|e^{-\frac{2(p+1) s}{p-1}} \mathscr{F}\left(e^{\frac{2 s}{p-1}}\left(K_{1}^{*}+q_{1}\right)\right)\right| \leq C e^{-\frac{p+1}{p-1} s}+\frac{C}{s^{\alpha}}\left(\left|K_{1}^{*}\right|^{p+1}+\left|q_{1}\right|^{p+1}\right) .
$$

Thanks to (ii) and (iv) in Lemma A.1 and (29), we conclude

$$
\int_{-1}^{1}\left|K_{1}^{*}\right|^{p+1} \rho d y \leq C \sum_{i=1}^{k} \int_{-1}^{1}\left|\kappa\left(d_{i}^{*}\right)\right|^{p+1} \rho d y \leq C .
$$


By integrating inequality (119) over $(-1,1)$, and taking into account (120), (29) and (i) in Lemma A.1, we obtain the estimate (117).

- Proceeding similarly as for (117) and using estimates (29) and (84), we easily deduce (118). This concludes the proof of Lemma A.2.

In order to construct a Lyapunov functional for equation (69), we need to prove the following estimates:

Lemma A.3. For all $s \in\left[s_{0}, \bar{s}\right)$, we have:

(i) (Control of the time derivative of $E_{1}$ )

$$
\frac{d}{d s} E_{1}(s) \leq-\frac{2}{p-1} \int_{-1}^{1} q_{2}^{2} \frac{\rho}{1-y^{2}} d y+C\left(\|q\|_{\mathscr{H}}+\bar{J}\right) \varphi(q, q)+C J \sqrt{\varphi(q, q)}+C J^{\bar{p}}+\frac{C}{s^{\alpha}} .
$$

(ii) (Control of the time derivative of $\int_{-1}^{1} q_{1} q_{2} \rho d y$ )

$$
\frac{d}{d s} \int_{-1}^{1} q_{1} q_{2} \rho d y \leq-\frac{7}{10} \varphi(q, q)+C \int_{-1}^{1} q_{2}^{2} \frac{\rho}{1-y^{2}} d y+C J \sqrt{\varphi(q, q)}+C J^{\bar{p}}+\frac{C}{s^{\alpha}} .
$$

Proof.

Proof of (i) (Control of the time derivative of the terms of $E_{1}$ ).

Using the definitions (114) of $\varphi$ and (71) of $\psi$, we write

$$
\left.\frac{1}{2} \frac{d}{d s} \varphi(q, q)=\varphi\left(\partial_{s} q, q\right)\right)+I_{4}(s)
$$

where

$$
I_{4}(s)=\frac{p(p-1)}{2} \sum_{j=1}^{k}(-1)^{j+1} \int_{-1}^{1}\left(d_{j}^{\prime} \partial_{d} \kappa_{1}^{*}+\nu_{j}^{\prime} \partial_{\nu} \kappa_{1}^{*}\right)\left|K_{1}^{*}\right|^{p-3} K_{1}^{*} q_{1}^{2} \rho d y .
$$

Using equation (69), we write

$$
\begin{aligned}
\varphi\left(\partial_{s} q, q\right)= & \varphi(\hat{L} q, q) \underbrace{-\sum_{j=1}^{k}(-1)^{j}\left[\left(\nu_{j}-\nu_{j}^{\prime}\right) \varphi\left(\partial_{\nu} \kappa^{*}, q\right)+d_{j}^{\prime} \varphi\left(\partial_{d} \kappa^{*}, q\right)\right]}_{I_{5}(s)} \\
& +\varphi((0, R), q)+\int_{-1}^{1} q_{2} \tilde{g}\left(q_{1}\right) \rho d y+\int_{-1}^{1} q_{2}\left(h\left(q_{1}\right)+\tilde{f}\left(q_{1}\right)\right) \rho d y .
\end{aligned}
$$

In the remaining part of the proof, we need some estimates proved in [52, Appendix C]. For that reason, we will recall the following estimates (C.24) and (C.26) from that 
paper and (C.39) in [51, Appendix C], true under hypothesis (29)

$$
\begin{aligned}
& \varphi(\hat{L} q, q)=-\frac{4}{p-1} \int_{-1}^{1} q_{2}^{2} \frac{\rho}{1-y^{2}} d y, \\
& \int_{-1}^{1} \kappa\left(d_{i}^{*}, y\right)\left|f\left(q_{1}\right)\right| \rho d y \leq C\|q\|_{\mathscr{H}}^{2}, \quad\left|R_{-}\right| \leq C\|q\|_{\mathscr{H}}^{\bar{P}+1}, \\
& \left.\left|\int_{-1}^{1} \partial_{d} \kappa_{1}^{*}\left(d_{i}, \nu_{i}\right)\right| K_{1}^{*}\right|^{p-3} K_{1}^{*} q_{1}^{2} \rho d y \mid \leq \frac{C}{1-d_{i}^{* 2}}\|q\|_{\mathscr{H}}^{2}, \\
& \left.\left|\int_{-1}^{1} \partial_{\nu} \kappa_{1}^{*}\left(d_{i}, \nu_{i}\right)\right| K_{1}^{*}\right|^{p-3} K_{1}^{*} q_{1}^{2} \rho d y \mid \leq \frac{C}{1-d_{i}^{* 2}}\|q\|_{\mathscr{H}}^{2}, \\
& \int_{-1}^{1} R^{2}\left(1-y^{2}\right) \rho d y \leq C\left(\hat{J}^{*}\right)^{2},
\end{aligned}
$$

where $\bar{p}$ is defined (34). Since we have from (84) and (85) $\hat{J}^{*} \leq C\left(J^{*}\right)^{\frac{\bar{p}}{2}} \leq C J^{\frac{\bar{p}}{2}}$, we get from (126)

$$
\begin{aligned}
\varphi((0, R), q)=\int_{-1}^{1} R q_{2} \rho d y & \leq \frac{1}{p-1} \int_{-1}^{1} q_{2}^{2} \frac{\rho}{1-y^{2}} d y+C \int_{-1}^{1} R^{2}\left(1-y^{2}\right) \rho d y \\
& \leq \frac{1}{p-1} \int_{-1}^{1} q_{2}^{2} \frac{\rho}{1-y^{2}} d y+C J^{\bar{p}} .
\end{aligned}
$$

Using (126), (115), (29), (84), (31) and (32), we write

$$
\left|I_{4}(s)\right|+\left|I_{5}(s)\right| \leq C\|q\|_{\mathscr{H}}^{3}+C\|q\|_{\mathscr{H}} J+C\|q\|_{\mathscr{H}}^{2} \bar{J}+\frac{C}{s^{\alpha}} .
$$

Moreover, by using (99), we write

$$
\begin{aligned}
\left|\int_{-1}^{1} q_{2} \tilde{g}\left(q_{1}\right) \rho d y\right| \leq \quad & C e^{-s} \int_{-1}^{1}\left(\left|\partial_{y} q_{1}\right|+\left|q_{2}\right|+\left|q_{1}\right|+\left|q_{1}\right|^{\frac{p+1}{2}}\right)\left|q_{2}\right| \rho d y \\
& +C e^{-s} \int_{-1}^{1}\left(\left|\partial_{y} K_{1}^{*}\right|+\left|K_{1}^{*}\right|+\left|K_{1}^{*}\right|^{\frac{p+1}{2}}\right)\left|q_{2}\right| \rho d y \\
& +C e^{-s} \sum_{j=1}^{k}\left|\nu_{j}\right| \int_{-1}^{1}\left|\partial_{\nu} \kappa_{1}^{*}\left(d_{j}, \nu_{j}\right)\right|\left|q_{2}\right| \rho d y+C e^{-\frac{2 p s}{p-1}}
\end{aligned}
$$

Proceeding similarly as for $I_{1}(s), I_{2}(s)$ and $I_{3}(s)$ defined in (100), we get

$$
\left|\int_{-1}^{1} q_{2} \tilde{g}\left(q_{1}\right) \rho d y\right| \leq C e^{-s} \int_{-1}^{1} q_{2}^{2} \frac{\rho}{1-y^{2}} d y+C e^{-s} .
$$

Gathering the estimates (123), (125), (126), (127), (128) and (130), we get for $s_{0}$ large enough,

$$
\begin{aligned}
\frac{1}{2} \frac{d}{d s} \varphi(q, q) \leq & -\frac{2}{p-1} \int_{-1}^{1} q_{2}^{2} \frac{\rho}{1-y^{2}} d y+\int_{-1}^{1} q_{2}\left(h\left(q_{1}\right)+\tilde{f}\left(q_{1}\right)\right) \rho d y \\
& +C\|q\|_{\mathscr{H}}^{3}+C\|q\|_{\mathscr{H}} J+C\|q\|_{\mathscr{H}}^{2} \bar{J}+C J^{\bar{p}}+\frac{C}{s^{\alpha}} .
\end{aligned}
$$

Using the definition of $R_{-}$, given in (113), we write

$$
R_{-}^{\prime}(s)=-\int_{-1}^{1}\left(\partial_{s} q_{1}+\partial_{s} K_{1}^{*}\right)\left(h\left(q_{1}\right)+\tilde{f}\left(q_{1}\right)\right) \rho d y-\frac{2}{p-1} \int_{-1}^{1}\left(q_{1}+K_{1}^{*}\right) \tilde{f}\left(q_{1}\right) \rho d y
$$




$$
+\frac{2(p+1)}{p-1} e^{-\frac{2(p+1) s}{p-1}} \int_{-1}^{1} \mathscr{F}\left(e^{\frac{2 s}{p-1}}\left(K_{1}^{*}+q_{1}\right)\right) \rho d y-I_{4}(s),
$$

where $I_{4}(s)$ is defined in (124). By exploiting the identity (98), identity (132) becomes

$$
\begin{aligned}
R_{-}^{\prime}(s)= & -\int_{-1}^{1} q_{2}\left(h\left(q_{1}\right)+\tilde{f}\left(q_{1}\right)\right) \rho d y \underbrace{-\frac{2}{p-1} \int_{-1}^{1}\left(q_{1}+K_{1}^{*}\right) \tilde{f}\left(q_{1}\right) \rho d y}_{I_{6}(s)} \\
& +\underbrace{\frac{2(p+1)}{p-1} e^{-\frac{2(p+1) s}{p-1}} \int_{-1}^{1} \mathscr{F}\left(e^{\frac{2 s}{p-1}}\left(K_{1}^{*}+q_{1}\right)\right) \rho d y}_{I_{7}(s)} \\
& +\underbrace{\sum_{j=1}^{k}(-1)^{j} \nu_{j} \int_{-1}^{1} \partial_{\nu} \kappa_{1}^{*}\left[h\left(q_{1}\right)+\tilde{f}\left(q_{1}\right)\right] \rho d y}_{I_{8}(s)}-I_{4}(s) .
\end{aligned}
$$

Thanks to (117), (118) and (29), we write

$$
\left|I_{6}(s)\right|+\left|I_{7}(s)\right| \leq \frac{C}{s^{\alpha}} .
$$

Finally, it remains only to control the term $I_{8}(s)$. By exploiting $(\mathrm{v})$ in Lemma A.1, we write

$$
\left|I_{8}(s)\right| \leq C \sum_{j=1}^{k} \frac{\left|\nu_{j}\right|}{1-\left|d_{j}^{*}\right|} \int_{-1}^{1} \kappa\left(d_{j}^{*}\right)\left(\left|h\left(q_{1}\right)\right|+\left|\tilde{f}\left(q_{1}\right)\right|\right) \rho d y .
$$

Similarly as for (84), (95), (81) and (29), we easily prove that

$$
\left|I_{8}(s)\right| \leq C \sum_{j=1}^{k} \frac{\left|\nu_{j}\right|}{1-\left|d_{j}^{*}\right|}\left(\|q\|_{\mathscr{H}}^{2}+\frac{C}{s^{\alpha}}\right) \leq C\|q\|_{\mathscr{H}}^{2} \bar{J}+\frac{C}{s^{\alpha}} .
$$

Gathering the estimates (133), (134), (136), (128), (29) and (30), we get

$$
R_{-}^{\prime}(s) \leq-\int_{-1}^{1} q_{2}\left(h\left(q_{1}\right)+\tilde{f}\left(q_{1}\right)\right) \rho d y+C\|q\|_{\mathscr{H}}^{3}+C\|q\|\left\|_{\mathscr{H}} J+C\right\| q \|_{\mathscr{H}}^{2} \bar{J}+\frac{C}{s^{\alpha}} .
$$

Thanks to (131), (137) and the definition of $E_{1}(s)$, given in (112), we clearly have

$$
\frac{d}{d s} E_{1}(s) \leq-\frac{2}{p-1} \int_{-1}^{1} q_{2}^{2} \frac{\rho}{1-y^{2}} d y+C\left(\|q\|_{\mathscr{H}}^{3}+\|q\|_{\mathscr{H}} J+\|q\|_{\mathscr{H}}^{2} \bar{J}+J^{\bar{p}}\right)+\frac{C}{s^{\alpha}} .
$$

According to (29), we can see that $\|q\|_{\mathscr{H}}$ can be made small enough provided that $s_{0}$ is large enough. As a consequence of (115) and (138), we get the desired estimate (121). This ends the proof of (i) in Lemma A.3.

$$
\text { Proof of (ii): Control of the time derivative of } \int_{-1}^{1} q_{1} q_{2} \rho d y
$$


Using equation (69) and the identity (98), we write

$$
\begin{aligned}
& \frac{d}{d s} \int_{-1}^{1} q_{1} q_{2} \rho d y=\int_{-1}^{1} \partial_{s} q_{1} q_{2} \rho d y+\int_{-1}^{1} \partial_{s} q_{2} q_{1} \rho d y \\
= & \underbrace{-\sum_{j=1}^{k}(-1)^{j}\left(d_{j}^{\prime} \int_{-1}^{1} \partial_{d} \kappa^{*} \cdot\left(q_{2}, q_{1}\right) \rho d y+\left(\nu_{j}^{\prime}-\nu_{j}\right) \int_{-1}^{1} \partial_{\nu} \kappa^{*} \cdot\left(q_{2}, q_{1}\right) \rho d y\right)}_{I_{9}(s)} \\
& +\int_{-1}^{1} q_{2}^{2} \rho d y+\int_{-1}^{1} q_{1}\left(\mathscr{L} q_{1}+\psi q_{1}-\frac{p+3}{p-1} q_{2}-2 y \partial_{y} q_{2}+h\left(q_{1}\right)+\tilde{f}\left(q_{1}\right)+\tilde{g}\left(q_{1}\right)+R\right) \rho d y,
\end{aligned}
$$

where the dot "." stands for the usual inner product in $\mathbb{R}^{n}$.

Let us first recall the following estimates proved in pages 2904 and 2905 of [52]:

$$
\begin{aligned}
& \int_{-1}^{1} q_{2}^{2} \rho d y \leq \int_{-1}^{1} q_{2}^{2} \frac{\rho}{1-y^{2}} d y, \int_{-1}^{1} q_{1}\left(\mathscr{L} q_{1}+\psi q_{1}\right) \rho d y \leq-\varphi(q, q)+\int_{-1}^{1} q_{2}^{2} \frac{\rho}{1-y^{2}} d y \\
& \left|-\frac{p+3}{p-1} \int_{-1}^{1} q_{1} q_{2} \rho d y-2 \int_{-1}^{1} q_{1} \partial_{y} q_{2} \rho d y\right| \leq \frac{1}{10} \varphi(q, q)+C \int_{-1}^{1} q_{2}^{2} \frac{\rho}{1-y^{2}} d y, \\
& \left|\int_{-1}^{1} q_{1} h\left(q_{1}\right) \rho d y\right| \leq \frac{1}{10} \varphi(q, q) .
\end{aligned}
$$

Furthermore, by using the Hardy Sobolev inequality stated in item (i) of Lemma A.1. (115), (126) and (184), we conclude

$$
\int_{-1}^{1} q_{1} R \rho d y \leq \frac{1}{10} \varphi(q, q)+C J^{\bar{p}} .
$$

Moreover, arguing as in (95) and (105), we obtain

$$
\left|\int_{-1}^{1} q_{1} \tilde{f}\left(q_{1}\right) \rho d y\right| \leq \frac{C}{s^{\alpha}}, \quad\left|\int_{-1}^{1} q_{1} \tilde{g}\left(q_{1}\right) \rho d y\right| \leq C e^{-s} .
$$

Note also that, from (ii) and (v) in Lemma A.1, (29), (84), the Cauchy-Schwarz inequality, we write

$$
\left|I_{9}(s)\right| \leq C \sum_{j=1}^{k} \frac{\left|d_{j}^{\prime}\right|+\left|\nu_{j}^{\prime}-\nu_{j}\right|}{1-d_{j}^{2}}\|q\|_{\mathscr{H} .}
$$

By exploiting (139), (31) and (32), we deduce that

$$
\left|I_{9}(s)\right| \leq C\left(\|q\|_{\mathscr{H}}^{3}+\|q\|_{\mathscr{H}} J+\|q\|_{\mathscr{H}}^{2} \bar{J}+\frac{1}{s^{\alpha}}\right) .
$$

According to (29), we can see that $\bar{J}$ and $\|q\|_{\mathscr{H}}$ can be made small enough provided that $s_{0}$ is large enough. Consequently, collecting the above estimates and (115), we obtain the desired estimate (122), and this ends the proof of (ii) in Lemma A.3. 
Now, we are able to prove (33).

Proof of (33): Let $\eta \in(0,1)$. By exploiting (121) and (122) and the definition of $E_{2}(s)$, given in (112), we have

$$
\begin{aligned}
\frac{d}{d s} E_{2}(s)+\frac{\eta}{2} E_{2}(s) \leq & -\left(\frac{\eta}{5}-C\left(\|q\|_{\mathscr{H}}+\bar{J}\right)\right) \varphi(q, q)+\frac{\eta^{2}}{2} \int_{-1}^{1} q_{1} q_{2} \rho d y+\frac{\eta}{2} R_{-}(s) \\
& -\left(\frac{2}{p-1}-C \eta\right) \int_{-1}^{1} q_{2}^{2} \frac{\rho}{1-y^{2}} d y+C J \sqrt{\varphi(q, q)}+C J^{\bar{p}}+\frac{C}{s^{\alpha}} .
\end{aligned}
$$

Observing inequalities (116), (117), (115), (29) along with the Cauchy-Schwarz inequality yield

$$
\frac{\eta^{2}}{2} \int_{-1}^{1} q_{1} q_{2} \rho+\frac{\eta}{2} R_{-}(s) \leq C \eta^{2} \varphi(q, q)+C \eta s_{0}^{(1-\min (p, 2)) / 2} \varphi(q, q)+\frac{C}{s^{\alpha}} .
$$

Moreover, by (29), we easily deduce that

$$
C J \sqrt{\varphi(q, q)} \leq \frac{\eta}{10} \varphi(q, q)+\frac{C}{\eta} J^{2} \leq \frac{\eta}{10} \varphi(q, q)+\frac{C}{\eta} J^{\bar{p}} .
$$

From (141), (142), we get

$$
\begin{aligned}
\frac{d}{d s} E_{2}(s)+\frac{\eta}{2} E_{2}(s) \leq & -\left(\frac{\eta}{5}-C \eta s_{0}^{(1-\min (p, 2)) / 2}-C \eta^{2}-C\left(\|q\|_{\mathscr{H}}+\bar{J}\right)\right) \varphi(q, q) \\
& -\left(\frac{2}{p-1}-C \eta\right) \int_{-1}^{1} q_{2}^{2} \frac{\rho}{1-y^{2}} d y+\frac{C}{\eta} J^{\bar{p}}+\frac{C}{s^{\alpha}}
\end{aligned}
$$

Note that, once again, due to the fact that $s_{0}$ is large enough, and according to (29), we can consider $\|q\|_{\mathscr{H}}, s_{0}^{(1-\min (p, 2)) / 2}$ and $\bar{J}$ as small terms. Then, there exists $\eta_{0}>0$ such that, for all $\eta \in\left(0, \eta_{0}\right]$, we have

$$
\frac{d}{d s} E_{2}(s)+\frac{\eta}{2} E_{2}(s) \leq \frac{C}{\eta} J^{\bar{p}}+\frac{C}{s^{\alpha}} .
$$

By definition (30) of $J$, we write

$$
\left|\left(J^{\bar{p}}\right)^{\prime}\right|=\frac{2 \bar{p}}{p-1}\left|\sum_{j=1}^{k-1}\left(\zeta_{j+1}^{\prime}-\zeta_{j}^{\prime}\right) e^{-\frac{2}{p-1}\left(\zeta_{j+1}-\zeta_{j}\right)}\right| J^{\bar{p}-1} \leq \frac{4 \bar{p} J^{\bar{p}}}{p-1} \sum_{j=1}^{k}\left|\zeta_{j}^{\prime}\right| .
$$

Exploiting (32) and (29), we conclude that $\left|\zeta_{j}^{\prime}\right| \leq C s_{0}^{-\frac{1}{2}}$. Consequently, we have

$$
\left|\left(J^{\bar{p}}\right)^{\prime}\right| \leq C s_{0}^{-\frac{1}{2}} J^{\bar{p}}
$$

Since $s_{0}$ is large enough, then we can write

$$
\left|\left(J^{\bar{p}}\right)^{\prime}\right| \leq \frac{\eta}{4} J^{\bar{p}} .
$$

Let us introduce

$$
E_{3}(s)=E_{2}(s)-\frac{1}{\eta^{3}} J^{\bar{p}} .
$$


By using (145), (148) and (149), we are now in a position to get,

$$
\frac{d}{d s} E_{3}(s)+\frac{\eta}{2} E_{3}(s) \leq \frac{1}{\eta^{2}}\left(C \eta-\frac{1}{4}\right) J^{\bar{p}}+\frac{C}{s^{\alpha}}, \quad \forall s \geq s_{0}, \quad \forall \eta \in\left(0, \eta_{0}\right] .
$$

Then for $\eta_{0}$ small enough, we have

$$
\frac{d}{d s} E_{3}(s)+\frac{\eta}{2} E_{3}(s) \leq \frac{C}{s^{\alpha}}, \quad \forall s \geq s_{0}, \quad \forall \eta \in\left(0, \eta_{0}\right] .
$$

Integrating the last inequality and using the definition of $E_{3}(s)$, we conclude

$$
E_{2}(s) \leq E_{2}\left(s_{0}\right) e^{-\frac{\eta\left(s-s_{0}\right)}{2}}+\frac{1}{\eta^{3}} J^{\bar{p}}+\frac{C}{\eta s^{\alpha}}, \quad \forall s \geq s_{0} \quad \forall \eta \in\left(0, \eta_{0}\right] .
$$

Using the definition of $E_{2}(s)$ as in (112), the estimates (115), (116), (117) and (142), we conclude

$$
\begin{aligned}
\phi(q, q) \leq & C\left\|q\left(s_{0}\right)\right\|_{\mathscr{H}}^{2} e^{-\frac{\eta\left(s-s_{0}\right)}{2}}+\frac{1}{\eta^{3}} J^{\bar{p}}+\frac{C}{\eta s^{\alpha}} \\
& +\left(C \eta+C s_{0}^{(1-\min (p, 2)) / 2}\right) \phi(q, q), \quad \forall s \geq s_{0}, \quad \forall \eta \in\left(0, \eta_{0}\right] .
\end{aligned}
$$

The above inequality, used for $\eta$ small enough, together with (115) imply (33). This concludes the proof of Proposition 2.3 .

Acknowledgements. The first author would like to thank the Laboratoire Analyse Géométrie et Applications (LAGA) of the University Paris 13, where he was invited as a Visiting Professor in June 2018 and where part of this work was done. He is grateful to all the LAGA Faculty members for the hospitality and the stimulating atmosphere.

\section{References}

[1] S. Alinhac. Blowup for nonlinear hyperbolic equations, volume 17 of Progress in Nonlinear Differential Equations and their Applications. Birkhäuser Boston Inc., Boston, MA, 1995.

[2] S. Alinhac. A minicourse on global existence and blowup of classical solutions to multidimensional quasilinear wave equations. In Journées "Équations aux Dérivées Partielles" (Forges-les-Eaux, 2002), pages Exp. No. I, 33. Univ. Nantes, Nantes, 2002.

[3] A. Bressan. On the asymptotic shape of blow-up. Indiana Univ. Math. J., 39(4):947960, 1990.

[4] A. Bressan. Stable blow-up patterns. J. Differential Equations, 98(1):57-75, 1992.

[5] J. Bricmont and A. Kupiainen. Universality in blow-up for nonlinear heat equations. Nonlinearity, 7(2):539-575, 1994.

[6] L. A. Caffarelli and A. Friedman. Differentiability of the blow-up curve for onedimensional nonlinear wave equations. Arch. Rational Mech. Anal., 91(1):83-98, 1985. 
[7] L. A. Caffarelli and A. Friedman. The blow-up boundary for nonlinear wave equations. Trans. Amer. Math. Soc., 297(1):223-241, 1986.

[8] J. Carr and R. L. Pego. Metastable patterns in solutions of $u_{t}=\varepsilon^{2} u_{x x}-f(u)$. Comm. Pure Appl. Math. 42:523-576, 1989.

[9] R. Côte. Construction of solutions to the subcritical gKdV equations with a given asymptotical behavior. J. Funct. Anal., 241(1):143-211, 2006.

[10] R. Côte. Construction of solutions to the $L^{2}$-critical KdV equation with a given asymptotic behaviour. Duke Math. J., 138(3):487-531, 2007.

[11] R. Côte, Y. Martel, and F. Merle. Construction of multi-soliton solutions for the $L^{2}$ supercritical gKdV and NLS equations. Rev. Mat. Iberoamericana, 27(1):273-302, 2011.

[12] R. Côte and H. Zaag. Construction of a multisoliton blowup solution to the semilinear wave equation in one space dimension. Comm. Pure Appl. Math., 66(10):15411581, 2013.

[13] R. Donninger, W. Schlag, and A. Soffer. On pointwise decay of linear waves on a Schwarzschild black hole background. Comm. Math. Phys., 309(1):51-86, 2012.

[14] G. K. Duong, V. T. Nguyen, and H. Zaag. Construction of a stable blowup solution with a prescribed behavior for a non-scaling invariant semilinear heat equation. Tunisian Journal of Mathematics, 1(1):13-45, 2019.

[15] M. A. Ebde and H. Zaag. Construction and stability of a blow up solution for a nonlinear heat equation with a gradient term. Bol. Soc. Esp. Mat. Apl, (55):5-21, 2011.

[16] V. Georgiev, and G. Todorova. Existence of a solution of the wave equation with nonlinear damping and source terms. J. Differential Equations., 109(2): 295-308, 1994.

[17] T. Ghoul, S. Ibrahim, and V. T. Nguyen. Construction of type II blowup solutions for the 1-corotational energy supercritical wave maps. J. Differential Equations., 265(7): 2968-3047, 2018.

[18] T. Ghoul and N. Masmoudi. Stability of infinite time aggregation for the critical patlak-keller-segel model in 2 dimension. 2016. submitted.

[19] T. Ghoul, V. T. Nguyen, and H. Zaag. Blowup solutions for a nonlinear heat equation involving a critical power nonlinear gradient term. J. Differential Equations, 263 (8):4517 - 4564, 2017.

[20] T. Ghoul, V. T. Nguyen and H. Zaag. Construction and stability of blowup solutions for a non-variational semilinear parabolic system. Annales de l'Institut Henri Poincaré C, Analyse non linéaire, 35 (6):1577-1630, 2018.

[21] T. Ghoul, V. T. Nguyen and H. Zaag. Blowup solutions for a reaction-diffusion system with exponential nonlinearities. J. Differential Equations, 264:7523-7579, 2018. 
[22] T. Ghoul, V. T. Nguyen and H. Zaag. Construction and stability of type I blowup solutions for non-variational semilinear parabolic systems. Proceedings of the Tunisian Mathematical Society Conference, Tabarka, Tunisia, 2018.

[23] T. Ghoul, V. T. Nguyen and H. Zaag. Construction of type I blowup solutions for a higher order semilinear parabolic equation. 2018. submitted.

[24] V. T. Nguyen and H. Zaag. Construction of a stable blow-up solution for a class of strongly perturbed semilinear heat equations. Ann. Sc. Norm. Super. Pisa Cl. Sci. 5., 16:1275-1314, 2016.

[25] M.A. Hamza, The blow-up rate for strongly perturbed semilinear wave equations in the conformal regime without a radial assumption. Asymptotic Analysis, 97, (3-4): 351-378, 2016.

[26] M.A. Hamza and O. Saidi, The blow-up rate for strongly perturbed semilinear wave equations. J. Dyn. Diff. Equ., 26: 1115-1131, 2014.

[27] M.A. Hamza and O. Saidi, The blow-up rate for strongly perturbed semilinear wave equations in the conformal case, Math Phys Anal Geom, 18(1)), Art. 15, 2015.

[28] M.A. Hamza and H. Zaag. A Lyapunov functional and blow-up results for a class of perturbed semilinear wave equations. Nonlinearity, 25(9):2759-2773, 2012.

[29] M.A. Hamza and H. Zaag. A Lyapunov functional and blow-up results for a class of perturbations for semilinear wave equations in the critical case. J. Hyperbolic Differ. Equ., 9:195-221, 2012.

[30] M. A. Hamza and H. Zaag. Blow-up behavior for the Klein-Gordon and other perturbed semilinear wave equations. Bull. Sci. Math., 137(8):1087-1109, 2013.

[31] M.A. Hamza and H. Zaag. Blow-up results for semilinear wave equations in the super-conformal case. Discrete Contin. Dyn. Syst. Ser. B, 18(9):2315-2329, 2013.

[32] S. Kichenassamy and W. Littman. Blow-up surfaces for nonlinear wave equations. I. Comm. Partial Differential Equations. 18 (3-4): 431-452, 1993.

[33] S. Kichenassamy and W. Littman. Blow-up surfaces for nonlinear wave equations. II. Comm. Partial Differential Equations. 18(11): 1869-1899, 1993.

[34] H. A. Levine. Instability and nonexistence of global solutions to nonlinear wave equations of the form $P u_{t t}=-A u+\mathscr{F}(u)$. Trans. Amer. Math. Soc., 192:1-21, 1974.

[35] H. A. Levine. Some additional remarks on the non-existence of global solution to non-linear wave equation. SIAM J. Math. Anal. 5:138-146,1974.

[36] H. A. Levine and G. Todorova. Blow up of solutions of the Cauchy problem for a wave equation with nonlinear damping and source terms and positive initial energy. SIAM J. Math. Anal. 5(3):793-805, 2001.

[37] H. Lindblad and C. D. Sogge. On existence and scattering with minimal regularity for semilinear wave equations. J. Funct. Anal. 130 (2), 357-426, 1995. 
[38] F. Mahmoudi, N. Nouaili, and H. Zaag. Construction of a stable periodic solution to a semilinear heat equation with a prescribed profile. Nonlinear Anal., 131:300-324, 2016.

[39] Y. Martel. Asymptotic $N$-soliton-like solutions of the subcritical and critical generalized Korteweg-de Vries equations. Amer. J. Math., 127(5):1103-1140, 2005.

[40] Y. Martel and F. Merle. Multi solitary waves for nonlinear Schrödinger equations. Ann. Inst. H. Poincaré Anal. Non Linéaire, 23(6):849-864, 2006.

[41] N. Masmoudi and H. Zaag. Blow-up profile for the complex Ginzburg-Landau equation. J. Funct. Anal., 255(7):1613-1666, 2008.

[42] F. Merle. Construction of solutions with exactly $k$ blow-up points for the Schrödinger equation with critical nonlinearity. Comm. Math. Phys., 129(2):223-240, 1990.

[43] F. Merle. Solution of a nonlinear heat equation with arbitrarily given blow-up points. Comm. Pure Appl. Math., 45(3):263-300, 1992.

[44] F. Merle, P. Raphaël, and I. Rodnianski. Blowup dynamics for smooth data equivariant solutions to the critical Schrödinger map problem. Invent. Math., 193(2):249$365,2013$.

[45] F. Merle and H. Zaag. Stabilité du profil à l'explosion pour les équations du type $u_{t}=\Delta u+|u|^{p-1} u$. C. R. Acad. Sci. Paris Sér. I Math., 322(4):345-350, 1996.

[46] F. Merle and H. Zaag. Stability of the blow-up profile for equations of the type $u_{t}=\Delta u+|u|^{p-1} u$. Duke Math. J., 86(1):143-195, 1997.

[47] F. Merle and H. Zaag. Existence and universality of the blow-up profile for the semilinear wave equation in one space dimension. J. Funct. Anal., 253(1):43-121, 2007.

[48] F. Merle and H. Zaag. Openness of the set of non characteristic points and regularity of the blow-up curve for the $1 \mathrm{~d}$ semilinear wave equation. Comm. Math. Phys., 282:55-86, 2008.

[49] F. Merle and H. Zaag. Isolatedness of characteristic points for a semilinear wave equation in one space dimension. In Séminaire sur les Équations aux Dérivées Partielles, 2009-2010, pages Exp. No. 11, 10p. École Polytech., Palaiseau, 2010.

[50] F. Merle and H. Zaag. Blow-up behavior outside the origin for a semilinear wave equation in the radial case. Bull. Sci. Math., 135(4):353-373, 2011.

[51] F. Merle and H. Zaag. Existence and classification of characteristic points at blow-up for a semilinear wave equation in one space dimension. Amer. J. Math., 134(3):581$648,2012$.

[52] F. Merle and H. Zaag. Isolatedness of characteristic points for a semilinear wave equation in one space dimension. Duke Math. J., 161(15):2837-2908, 2012.

[53] V. T. Nguyen and H. Zaag. Construction of a stable blow-up solution for a class of strongly perturbed semilinear heat equations. Ann. Sc. Norm. Super. Pisa Cl. Sci. 5., 16:1275-1314, 2016. 
[54] N. Nouaili and H. Zaag. Profile for a Simultaneously Blowing up Solution to a Complex Valued Semilinear Heat Equation. Comm. Partial Differential Equations, 40(7):1197-1217, 2015.

[55] N. Nouaili and H. Zaag. Construction of a blow-up solution for the Complex Ginzburg-Landau equation in some critical case. Arch. Ration. Mech. Anal., to appear, 2018.

[56] P. Raphaël and R. Schweyer. On the stability of critical chemotactic aggregation. Math. Ann., 359(1-2):267-377, 2014.

[57] R. Schweyer. Type II blow-up for the four dimensional energy critical semi linear heat equation. J. Funct. Anal., 263(12):3922-3983, 2012.

[58] S. Tayachi and H. Zaag. Existence of a stable blow-up profile for the nonlinear heat equation with a critical power nonlinear gradient term. Trans. Amer. Math. Soc, to appear 2018.

[59] S. Tayachi and H. Zaag. Existence of a stable blow-up profile for the nonlinear heat equation with a critical power nonlinear gradient term. In C. Dogbe, editor, Actes du colloque EDP-Normandie, pages 119-136, 2015.

[60] G. Todorova. Cauchy problem for a non linear wave equation with non linear damping and source terms. Nonlinear Anal., 891-905, 2000.

[61] G. B. Whitham. Linear and nonlinear waves. Pure and Applied Mathematics (New York), John Wiley \& Sons Inc., New York, 1999. Reprint of the 1974 original, A Wiley-Interscience Publication.

[62] H. Zaag. Blow-up results for vector-valued nonlinear heat equations with no gradient structure. Ann. Inst. H. Poincaré Anal. Non Linéaire, 15(5):581-622, 1998.

\section{Address:}

Imam Abdulrahman Bin Faisal University P.O. Box 1982 Dammam, Saudi Arabia.

e-mail: mahamza@iau.edu.sa

Université Paris 13, Institut Galilée, Laboratoire Analyse, Géométrie et Applications, CNRS UMR 7539, 99 avenue J.B. Clément, 93430 Villetaneuse, France.

e-mail: Hatem.Zaag@univ-paris13.fr 